\title{
Large-eddy simulation and multiscale modelling of a Richtmyer-Meshkov instability with reshock
}

\author{
By D. J. HILL, C. PANTANO AND D. I. PULLIN \\ Graduate Aeronautical Laboratories, California Institute of Technology, Pasadena, CA 91125, USA \\ dh@galcit.caltech.edu
}

(Received 6 July 2005 and in revised form 4 November 2005)

Large-eddy simulations of the Richtmyer-Meshkov instability with reshock are presented and the results are compared with experiments. Several configurations of shocks initially travelling from light (air) to heavy (sulfur hexafluoride, $\mathrm{SF}_{6}$ ) have been simulated to match previous experiments and good agreement is found in the growth rates of the turbulent mixing zone (TMZ). The stretched-vortex subgrid model used in this study allows for subgrid continuation modelling, where statistics of the unresolved scales of the flow are estimated. In particular, this multiscale modelling allows the anisotropy of the flow to be extended to the dissipation scale, $\eta$, and estimates to be formed for the subgrid probability density function of the mixture fraction of air/ $\mathrm{SF}_{6}$ based on the subgrid variance, including the effect of Schmidt number.

\section{Introduction}

During the process of refraction as a shock interacts with an interface separating two gases, vorticity is, in general, deposited at the interface by means of baroclinic torque. The physical mechanism of this vorticity production is the miss-alignment of the pressure gradient across the shock and the local density gradient at the contact during shock passage. The rotational flow associated with this localized vorticity causes the interface to distort strongly in areas of maximal misalignment, forming characteristic structures such as bubbles and spikes. The growth in the interface amplitude resulting from the shock-interface interaction is generally referred to as the Richtmyer-Meshkov instability (RMI) (Richtmyer 1960; Meshkov 1969) and is sometimes considered to be the impulsive limit of a Rayleigh-Taylor instability. The analysis of Richtmyer (1960) showed that initially small perturbation amplitudes grow linearly with time, and other work such as Mikaelian (1989) suggests that even when the perturbation is nonlinearly saturated, the growth continues to be linear in time. The structures generated by the Richtmyer-Meshkov instability are themselves subject to vortex pairing and additional instabilities such as the Kelvin-Helmholtz instability, leading to a wide range of physical scales in the area of the interface.

The Richtmyer-Meshkov instability plays a fundamental role in the context of many physical settings, both natural and man-made. Evidence of the RMI has been seen by the Hubble Space Telescope in remnants of the explosion of supernova 1987A (Sonneborn et al. 1999; Maran et al. 2000). In related stellar events, the RMI is used to explain the overturn of the outer portion of collapsing cores of supernovas (Smarr et al. 1981). Proposals have been made to exploit the mixing properties of the instability in supersonic combustion engines (Yang, Kubota \& Zukoski 1993). Conversely, in the context of inertial confinement fusion, the RMI-induced mixing between fuel and capsule is a liability which provides considerable challenges in 
achieving the required compression (Lindl, McCrory \& Campbell 1992). This last example alone has motivated considerable interest in the fundamental aspects of the RMI; recent reviews of the RMI include Zabusky (1999) and Brouillette (2002).

This paper examines, by computational simulation, a canonical RichtmyerMeshkov instability realized within the confines of a shock-tube as a planar shock interacts with a co-planar density interface formed by the contact between two gases, air and sulfur hexafluoride $\left(\mathrm{SH}_{6}\right)$ (Vetter \& Sturtevant 1995). Slight perturbations or irregularities in the density interface, for example local deviations from co-planarity, form the density misalignments required to initiate RMI during shock interaction. A second and much more energetic RMI occurs after the initial shock has traversed the extent of the shock-tube, reflected off the tube end and reshocks the now greatly distorted interface.

The double-shock process produces a large dynamical range of turbulent scales, requiring, with presently available computational resources, the techniques of large-eddy simulation (LES). This approach entails a loss of detailed information contained in fine unresolved scales whose dynamical interaction with the computationally resolved scales is only modelled. Because mixing of the two gases in the turbulent zone is an essentially small-scale (subgrid) process, conventional LES, with its exclusive focus on resolved-scale transport, cannot accurately capture this important aspect of the flow. Owing to its structural ansatz, using vortices that are local (asymptotic) solutions to the Navier-Stokes equations at the subgrid level, the stretched-vortex model enables estimation of the contribution to certain statistical quantities from scales below the resolved-scale cutoff. This, together with resolved-scale information, allows some degree of truly multiscale modelling of the flow, including predictions of mixing.

Apart from the physical modelling issues associated with LES, simulation of compressible turbulent flows demands two mutually exclusive numerical approaches. On one hand, the presence of shocks in three-dimensional flows, whose length scale is of the order of the mean free path, implies that the numerical method must be of a shockcapturing type. On the other hand, turbulence is better simulated when the numerical method is non-dissipative. Since all shock-capturing methods are dissipative, two mutually orthogonal numerical requirements arise. To address this difficulty, we developed hybrid numerical methods that are shock-capturing around discontinuities (shocks) and revert to centred non-dissipative discretizations in the remaining regions of the flow, including those encompassing the regions of turbulent activity. The present method is an extension of the hybrid centred-upwinded algorithm of Hill \& Pullin (2004).

The target experiment of Vetter \& Sturtevant (1995) and the modelling of the laboratory initial and boundary conditions used at present are discussed in $\S 2$. There follows, in $\S 3$, a short description of the relevant filtered Navier-Stokes equations and the stretched-vortex subgrid-scale (SGS) model used in the simulations. In $\S 4$, we give an account of the hybrid numerical scheme employed. The main resolved-scale simulation results are presented in $\S 5$. The multiscale modelling based on subgrid continuation is described in $\S \S 6$ and 7. This includes predictions of subgrid mixing properties and estimates of the effect of Schmidt number on both scalar spectra and scalar probability density functions (p.d.f.).

\section{Flow description}

We discuss, in the following order: the experimental flow conditions, simulation boundary conditions, the treatment of the sidewalls of the shock-tube, the endwall of 


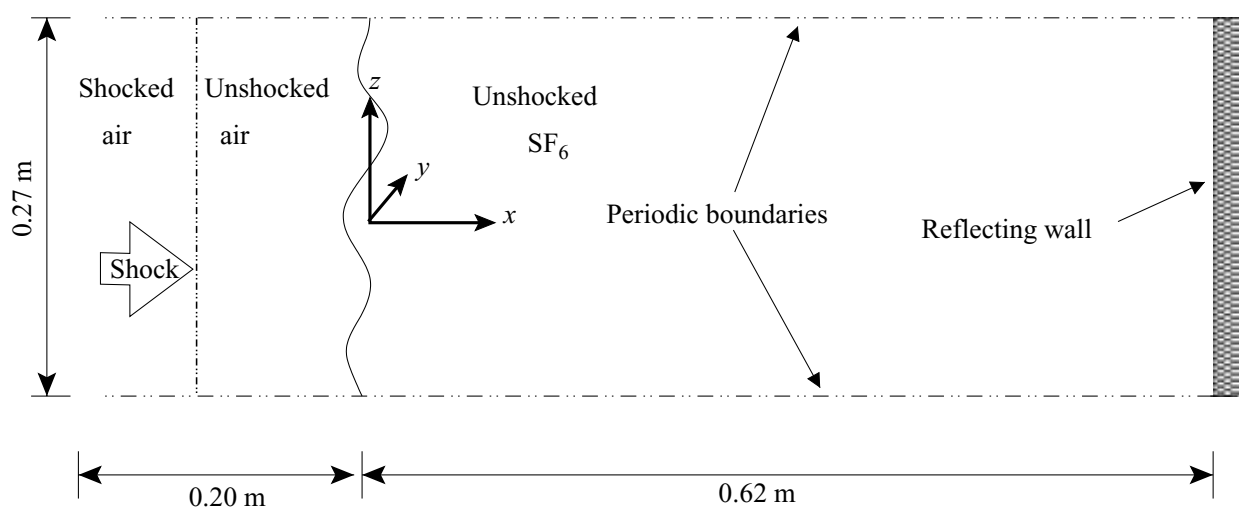

FIGURE 1. Geometry of the simulation domain for the Mach 1.50 case, indicating the location of the mathematical origin relative to the reflecting endwall. Note the full simulation domain is square in $(y, z)$-cross-section with periodic boundary conditions also applied at $y, z= \pm 0.135 \mathrm{~m}$.

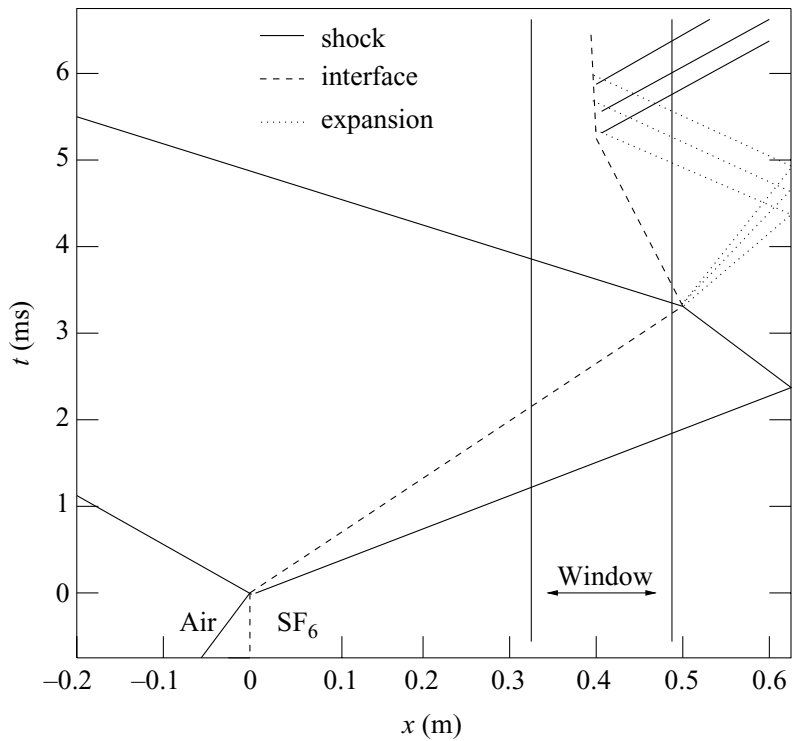

FiguRE 2. Approximate wave diagram for the interaction of a $M=1.50$ shock with the air $/ \mathrm{SF}_{6}$ interface. Both the exit of the initial reflected shock and the primary shock are indicated, as is the field of view for the flow-visualization system.

the shock tube and the open end of the tube and, finally, the initial configuration. A schematic of the initial flow configuration, including the incident shock, the perturbed interface between the two test gases, and other features of the geometry and boundary conditions, is shown in figure 1 . Figure 2 shows a one-dimensional wave-diagram of the RMI in an $(x, t)$-plane, including the first interaction of the shock with the interface, shock reflection off the tube endwall, reshock, and the interaction of a reflected expansion (resulting from reshock) with the gas interface.

\subsection{Experimental domain and parameters}

For the purpose of validation, we have chosen for numerical investigation the air/ sulfur hexafluoride $\left(\mathrm{SF}_{6}\right)$ Richtmyer-Meshkov variable-length shock-tube experiments 
Incident Mach number

Pressure $(\mathrm{kPa})$

Length, membrane to endwall $(\mathrm{cm})$

Instantaneous velocity $\left(\mathrm{m} \mathrm{s}^{-1}\right)$

Shocked growth rate $\left(\mathrm{m} \mathrm{s}^{-1}\right)$

Reshocked growth rate $\left(\mathrm{m} \mathrm{s}^{-1}\right)$

Shocked observation times (ms)

Reshocked observation times (ms)

$\begin{array}{ccc}\mathrm{II}^{b} & \mathrm{VI}^{b} & \mathrm{VII}^{b} \\ 1.24 & 1.50 & 1.98 \\ 40 & 23 & 8 \\ 110 & 62 & 49 \\ 72 & 150 & 287 \\ 2.1 & 4.2 & 7.5 \\ 17.0 & 37.2 & 74.4 \\ 4.7-6.7 & 2.2-3.2 & 1.7-2.5 \\ 15.5-16.5 & 4.0-5.5 & 1.7-2.5\end{array}$

TABLE 1. The test conditions and growth rates of the interface thickness from Vetter \& Sturtevant (1995). These experiments correspond to $\mathrm{II}^{b}, \mathrm{VI}^{b}$, and $\mathrm{VII}^{b}$ therein and the observation times are approximate as they result from figure 6 of that work.

$\begin{array}{lcr}\text { Property } & \text { Air } & \mathrm{SF}_{6} \\ \text { Molecular mass }\left(\mathrm{kg} \mathrm{kmol}^{-1}\right) & 29.04 & 146.07 \\ \text { Ratio of specific heats: } \gamma & 1.40 & 1.09 \\ \text { Density }\left(\mathrm{kg} \mathrm{m}^{-3}\right) & 1.18 & 5.97 \\ \text { Kinematic viscosity }\left(10^{-6} \mathrm{~m}^{-2} \mathrm{~s}^{-1}\right) & 15.7 & 2.47 \\ \text { Prandtl number } & 0.71 & 0.90 \\ \text { Diffusion coefficient in air }\left(10^{-6} \mathrm{~m}^{-2} \mathrm{~s}^{-1}\right) & 20.4 & 9.7\end{array}$

TABLE 2. Gas properties of air and $\mathrm{SF}_{6}$ at $25^{\circ} \mathrm{C}$ and $1 \mathrm{~atm}$, as used in the simulation.

of Vetter \& Sturtevant (1995). In these experiments, a shock in air travelled down the tube with a Mach number strength ranging from 1.18 to 1.98. The shock encountered a contact surface in the form of a thin membrane interface separating the air from the remainder of the tube filled with $\mathrm{SF}_{6}$. The passage of the transmitted shock induced a mixing zone between the air and $\mathrm{SF}_{6}$ which was instantaneously accelerated to reported velocities of $56 \mathrm{~m} \mathrm{~s}^{-1}$ to $287 \mathrm{~m} \mathrm{~s}^{-1}$, depending on the case. Using either sparkschlieren photography or high-speed motion pictures, data were recorded as the mixing zone passed the field of view of an observation port, and data were again recorded after the transmitted shock reflected off the closed end of the shock tube and 'reshocked' the mixing zone, bringing the mixing zone back into the observation field and reducing its mean velocity substantially. The length of the shock tube from the interface to the endwall was adjusted prior to each experiment to ensure that the reshocked mixing zone would develop in view. Vetter \& Sturtevant (1995) measured the instantaneous width of the mixing zone and calculated two mixing-zone growth rates, one after the initial shock and a second following reshock, for each experiment. These growth rates serve as the primary comparison between the experiments and the present simulations.

Owing to diagnostic limitations, the experiments with single-spark schlieren allowed for very few measurements of the mixing-layer width as the mixing zone passed the observation port. For this reason, the comparisons in this paper focus on three of the experiments in which high-speed motion picture photography was used, as the growth rates are ostensibly more accurate. These experiments correspond to Mach numbers $1.24,1.50$ and 1.98 and are referred to as cases $\mathrm{II}^{b}, \mathrm{VI}^{b}$ and $\mathrm{VII}^{b}$, respectively. Table 1 summarizes the experimental configurations and results. In the LES, we match the physical dimensions of the experimental domain and the particular gas properties of air and $\mathrm{SF}_{6}$, as summarized in table 2. The shock tube itself was square in crosssection with dimensions $0.27 \mathrm{~m} \times 0.27 \mathrm{~m}$ and of variable down-tube length. More 
precisely, in right-handed Cartesian coordinates, $\boldsymbol{x}=\left(x_{1}, x_{2}, x_{3}\right)=(x, y, z)$, the positive $x$-axis aligns with the down-tube direction with $x=0$ at the initial gas interface towards the reflecting wall. The simulation domain was $-0.20 \mathrm{~m} \leqslant x \leqslant L \times-0.135 \mathrm{~m} \leqslant$ $y \leqslant 0.135 \mathrm{~m} \times-0.135 \mathrm{~m} \leqslant z \leqslant 0.135 \mathrm{~m}$ where $L$ is $1.10 \mathrm{~m}, 0.62 \mathrm{~m}$ or $0.49 \mathrm{~m}$ for different runs. A small portion of the tube with $x<0$ is included in the computation, so that reflected and transmitted shocks will be essentially planar when they exit the domain.

\subsection{Modelling boundary conditions}

To facilitate interpretation of statistical results, the sidewalls of the shock tube in the simulations were replaced with periodic boundary conditions, thus allowing the calculation of planar spectra in quantities of interest and providing two statistically homogeneous directions. In making such a boundary approximation, the non-uniform boundary layer flow at the physical walls is absent from the simulation, but this is not found to be critical in comparing with the experiment. In fact, because such boundary effects lead to ambiguities in the interpretation of flow-visualization photographs, Vetter \& Sturtevant (1995) explicitly designed their experiment to ensure that the shock-wave/boundary-layer interaction would not play a dominant role in the measured growth rates.

The endwall of the shock tube (located at $x=L$ ) is, computationally, a reflecting boundary. No wall model or other special treatment was required in the large-eddy simulations at the reflecting end of the tube. The measured widths and approximate locations of the mixing zone suggested that the turbulent region does not reach the reflecting wall, an assumption which was born out in our simulations. In the computational domain, the shock tube is truncated with an open end. The treatment of this open end models the effects of the unsimulated remainder of the physical tube. To allow for stable long-time integration after the reflected shock has exited the computational domain, the density, velocity, and pressure are prescribed in the form of characteristic boundary conditions (Thompson 1987) at the exit plane.

\subsection{Modelling initial conditions}

The flow is initialized in three regions distributed from left to right: a post-shock state in the air side consistent with a shock travelling at the corresponding Mach number in the positive direction towards the contact between the two gases, a region of unshocked stationary air, and finally unshocked stationary $\mathrm{SF}_{6}$. The quiescent gases are assumed to be in thermal equilibrium and under uniform pressure. In agreement with the experiments, this initial pressure is taken, for each case, to be $40 \mathrm{kPa}, 23 \mathrm{kPa}$, or $8 \mathrm{kPa}$ (see table 1) and a room temperature of $286^{\circ} \mathrm{K}$ was assumed. Taken together, these choices determine the complete state of the unshocked gases. A schematic of the initial flow configuration, including the incident shock, the perturbed interface between the two test gases, and other features of the geometry and boundary conditions, is shown in figure 1.

The shock is initialized at a location of $-0.05 \mathrm{~m}$ in the $x$-direction and the lateral centroid of the perturbed contact at $x=0.0 \mathrm{~m}$ leading to an initial shock interaction which instantaneously accelerates the contact. Vetter \& Sturtevant (1995) indicate that a relatively weak expansion wave from the driver section of the shock tube follows, $0.5 \mathrm{~ms}$ in the Mach 1.5 case, after the shock. In single-mode Mach 1.5 experiments with the same shock tube and gases Prasad et al. (2000) also observe this expansion and indicate that its amplitude was sufficiently small as to be ignored in the presentation of their results. In light of both this and the fact that insufficient details were presented for the cases of interest, this expansion wave was neglected in the initial conditions for our simulations. Also, no attempt was made to model the effects of the composition 
of the thin $(0.5 \mu \mathrm{m})$ nitrocellulose membrane, but the shape was represented as a perturbed interface. These perturbations result from the wire mesh that was installed to support the membrane and they provide spatial variations in density which in turn give rise to the Richtmyer-Meshkov instability. The wire mesh formed a regular grid with spacing of $1 \mathrm{~cm}$ in both the horizontal and vertical directions (the wire diameter was $0.23 \mathrm{~mm}$ ). Prior to each experiment, the membrane was pushed into the mesh with an estimated amplitude of about $1 \mathrm{~mm}$. Vetter \& Sturtevant (1995) showed that by varying the order of the vertical and horizontal meshes and the membrane, the pre-reshock growth rates could vary by an order of magnitude. It is unclear to what extent this is due to the additional mixing of the membrane or to the sensitivity to the initial interface shape. Additionally, Greenough \& Burke (2004) have demonstrated that the initial density perturbation spectrum can have a strong effect even on the nonlinear stages of mixing-layer growth. In light of the uncertainty in the initial conditions and the role of the membrane, the actual width of the mixing zone is not expected to compare well during the observation times prior to reshock.

An interface representation similar to that employed by Cohen et al. (2002) is used here to incorporate small modes, ostensibly produced by the wire mesh, and additional symmetry-breaking modes which model the more random smaller-scale irregularities. The simulation interface was taken to be the linear combination of a regular 'eggcarton' perturbation with a much smaller irregular perturbation. Mathematically, the interface was prescribed as follows:

$$
x_{I}(y, z)=a_{0}|\sin (\pi y / \lambda) \sin (\pi z / \lambda)|+a_{1} h(y, z),
$$

where the first term, the so-called 'egg-carton', $|\sin (\pi y / \lambda) \sin (\pi z / \lambda)|$, models the regularity of the mesh and the second, $h(y, z)$, takes the form of a symmetry breaking perturbation with random phase, but with a prescribed initial power spectra of the form $k^{4} \exp \left(-\left(k / k_{o}\right)^{2}\right)$. The function $h(y, z)$ was computed once and saved so that it could be used for all the runs with the same computational resolution.

The fundamental carton wavelength, $\lambda$, was taken to be $27 \mathrm{~m} / 14 \approx 0.02 \mathrm{~m}$ as a compromise between the actual grid spacing of $0.01 \mathrm{~m}$ and the desire to allow enough resolution so that each perturbation could evolve into the nonlinear regime. Cohen et al. (2002) found it necessary to strike a similar balance. In the non-symmetric portion $h(y, z)$, the parameter $k_{o}=4$ was chosen, which corresponds to a peak wavelength of $(\pi / \sqrt{8}) \mathrm{m}$, and values of the coefficients $a_{0}$ and $a_{1}$ were $0.25 \mathrm{~cm}$ and $0.025 \mathrm{~cm}$, respectively.

\section{Equations of motion and subgrid modelling}

\subsection{Two-component Favre-filtered Navier-Stokes equations}

The Favre-filtered (i.e. density weighted) Navier-Stokes equations provide a natural separation of the large scales to be simulated from the small scales to be modelled (Zang, Dahlburg \& Dahlburg 1992). We denote Favre-filtered quantities by

$$
\tilde{f}=\frac{\overline{\rho f}}{\bar{\rho}},
$$

where $f$ is an arbitrary field, $\rho$ is the density and the overbar indicates the filtering operation

$$
\bar{f}(\boldsymbol{x})=\int G\left(\boldsymbol{x}-\boldsymbol{x}^{\prime}\right) f\left(\boldsymbol{x}^{\prime}\right) \mathrm{d} x^{\prime},
$$


with convolution kernel $G$. The filtering procedure combined with some modelling assumptions (e.g. negligible subgrid viscous work and triple correlations) leads to the following LES equations of motion for the density $\bar{\rho}$, momentum $\bar{\rho} \tilde{u}_{i}$, total energy $\bar{E}$, and a scalar representing mixture fraction $\tilde{\psi}$. The dimensional conservation transport equations are

$$
\begin{aligned}
& \frac{\partial \bar{\rho}}{\partial t}+\frac{\partial \bar{\rho} \tilde{u}_{j}}{\partial x_{j}}=0, \\
& \frac{\partial \bar{\rho} \tilde{u}_{i}}{\partial t}+\frac{\partial\left(\bar{\rho} \tilde{u}_{i} \tilde{u}_{j}+\bar{p} \delta_{i j}\right)}{\partial x_{j}}=\frac{\partial d_{i j}}{\partial x_{j}}-\frac{\partial \tau_{i j}}{\partial x_{j}}, \\
& \frac{\partial \bar{E}}{\partial t}+\frac{\partial(\bar{E}+\bar{p}) \tilde{u}_{j}}{\partial x_{j}}=\frac{\partial}{\partial x_{j}}\left(\bar{\kappa} \frac{\partial \bar{T}}{\partial x_{j}}\right)+\frac{\partial d_{j i} \tilde{u}_{i}}{\partial x_{j}}-\frac{\partial q_{j}^{T}}{\partial x_{j}}, \\
& \frac{\partial \bar{\rho} \tilde{\psi}}{\partial t}+\frac{\partial\left(\bar{\rho} \tilde{\psi} \tilde{u}_{j}\right)}{\partial x_{j}}=\frac{\partial}{\partial x_{j}}\left(\bar{\rho} \tilde{D} \frac{\partial \tilde{\psi}}{\partial x_{j}}\right)-\frac{\partial q_{j}^{\psi}}{\partial x_{j}},
\end{aligned}
$$

where repeated indices denote summation and the subgrid terms

$$
\begin{aligned}
\tau_{i j} & =\bar{\rho}\left(\widetilde{u_{i} u_{j}}-\tilde{u}_{i} \tilde{u}_{j}\right), \\
q_{j}^{T} & =\bar{\rho}\left(\widetilde{c_{p} T u_{j}}-\tilde{c}_{p} \tilde{T} \tilde{u}_{j}\right), \\
q_{j}^{\psi} & =\bar{\rho}\left(\widetilde{\psi u_{j}}-\tilde{\psi} \tilde{u}_{j}\right),
\end{aligned}
$$

represent subgrid stress tensor, and the heat and scalar transport flux, respectively. The filtered total energy $\bar{E}$ contains the subgrid kinetic energy and is given by

$$
\bar{E}=\frac{\bar{p}}{(\tilde{\gamma}-1)}+\frac{1}{2} \bar{\rho}\left(\tilde{u}_{k} \tilde{u}_{k}\right)+\frac{1}{2} \tau_{k k}
$$

while the filtered pressure, $\bar{p}$, is determined from the ideal equation of state for a mixture of gases,

$$
\bar{p}=\frac{\bar{\rho} R \tilde{T}}{\tilde{m}},
$$

where $R$ is the ideal gas constant; we have neglected temperature-species composition correlations in (3.6). This equation, together with (3.5), defines the Favre-filtered temperature $\tilde{T}$ as a function of $\bar{E}$, kinetic energy and the composition of the mixture. As there are only two gases, the single scalar $\psi$ is sufficient to specify the local mixture composition. Formally, air is assumed to behave as a single species with the average molecular weight of air. Within these assumptions, $\psi$ takes the value 0 in the air side and 1 in the $\mathrm{SF}_{6}$ side. The mass fraction of air is $\chi_{a}=1-\psi$, likewise for $\mathrm{SF}_{6} \chi_{s}=\psi$. The mean molecular weight is then given by $1 / \tilde{m}=\left(\tilde{\chi}_{a} / m_{a}+\tilde{\chi}_{s} / m_{s}\right)$, where $m_{a}$ and $m_{s}$ denote the molecular weights of air and $\mathrm{SF}_{6}$, respectively.

Transport properties of the mixture are determined from binary mixing rules and the pure component transport properties (Reid, Pransuitz \& Poling 1987). In the case of viscosity $\bar{\mu}$ (similarly for heat conduction, $\bar{\kappa}$, and diffusivity, $\tilde{D}$ ), each gas obeys $\mu_{l}=\mu_{l}^{o}\left(\tilde{T} / T_{o}\right)^{0.786}$, with $l=a, s$ (air and $\mathrm{SF}_{6}$, respectively), then

$$
\bar{\mu}=\frac{\mu_{a} \tilde{\chi}_{a} m_{a}^{-1 / 2}+\mu_{s} \tilde{\chi}_{s} m_{s}^{-1 / 2}}{\tilde{\chi}_{a} m_{a}^{-1 / 2}+\tilde{\chi}_{s} m_{s}^{-1 / 2}} .
$$


The deviatoric Newtonian stress tensor $d_{i j}$ of the mixture with appropriately filtered quantities is then expressed as

$$
d_{i j}=\bar{\mu}\left(\left(\frac{\partial \tilde{u}_{i}}{\partial x_{j}}+\frac{\partial \tilde{u}_{j}}{\partial x_{i}}\right)-\frac{2}{3} \frac{\partial \tilde{u}_{k}}{\partial x_{k}} \delta_{i j}\right) .
$$

Finally, the average specific heat ratio is defined by

$$
\tilde{\gamma}=\frac{\tilde{c}_{p}}{\tilde{c}_{v}}
$$

where the specific heat capacity at constant pressure is given by

$$
\tilde{c}_{p}=c_{p, a} \tilde{\chi}_{a}+c_{p, s} \tilde{\chi}_{s}
$$

and $\tilde{c}_{v}=\tilde{c}_{p}-R / \tilde{m}$. The element heat capacities, $c_{p, l}$, are assumed to be independent of temperature in the present study.

The filtering procedure described in (3.2) is purely formal. It is not, and cannot be, performed in LES, unless we have the full DNS (or experimental) fields in hand, in which case LES is irrelevant. Here and hereinafter, we identify variables defined formally by (3.1) and (3.2) with resolved-scale quantities in actual LES. This is strictly a resolved-scale modelling assumption at the level of those for subgrid quantities described below. For a discussion of this and other conceptual foundations of LES, see Pope (2004).

\subsection{Application of the stretched-vortex subgrid model}

Closure of the LES equations is completed in the form of a model for the subgrid interaction terms: stress tensor, $\tau_{i j}$, turbulent temperature flux, $q_{i}^{T}$, and mixture fraction flux, $q_{i}^{\psi}$. This is achieved by using the stretched-vortex model, originally developed for incompressible LES by Misra \& Pullin (1997), but extended to compressible flows (Kosovic, Pullin \& Samtaney 2002) and subgrid scalar transport (Pullin 2000). In this model, the flow within a computational grid cell is assumed to result from an ensemble of straight, nearly axisymmetric vortices aligned with the local resolved scale strain or vorticity. The resulting subgrid stresses are

$$
\begin{aligned}
\tau_{i j} & =\bar{\rho} \tilde{k}\left(\delta_{i j}-e_{i}^{v} e_{j}^{v}\right), \\
q_{i}^{T} & =-\bar{\rho} \frac{\Delta_{c}}{2} \tilde{k}^{1 / 2}\left(\delta_{i j}-e_{i}^{v} e_{j}^{v}\right) \frac{\partial\left(\tilde{c}_{p} \tilde{T}\right)}{\partial x_{j}}, \\
q_{i}^{\psi} & =-\bar{\rho} \frac{\Delta_{c}}{2} \tilde{k}^{1 / 2}\left(\delta_{i j}-e_{i}^{v} e_{j}^{v}\right) \frac{\partial \tilde{\psi}}{\partial x_{j}},
\end{aligned}
$$

where $\tilde{k}=\int_{k_{c}}^{\infty} E(k) \mathrm{d} k$ is the subgrid energy, $\boldsymbol{e}^{v}$ is the unit vector aligned with the subgrid vortex axis, $v=\bar{\mu} / \bar{\rho}$ is the kinematic viscosity and $k_{c}=\pi / \Delta_{c}$ represents the largest resolved wavenumber. This subgrid turbulent kinetic energy, $\tilde{k}$, is estimated by assuming a spiral vortex of the Lundgren (1982) form, whose energy (velocity) spectrum for the subgrid motion is given by

$$
E(k)=K_{0} \epsilon^{2 / 3} k^{-5 / 3} \exp \left[-2 k^{2} v /(3|\tilde{a}|)\right],
$$

where $K_{0}$ is the Kolmogorov prefactor, $\epsilon$ is the local cell-averaged dissipation (resolved flow plus subgrid scale) and $\tilde{a}=\tilde{S}_{i j} e_{i}^{v} e_{j}^{v}$ is the axial strain along the subgrid vortex axis where

$$
\tilde{S}_{i j}=\frac{1}{2}\left(\frac{\partial \tilde{u}_{i}}{\partial x_{j}}+\frac{\partial \tilde{u}_{j}}{\partial x_{i}}\right),
$$

denotes the resolved rate-of-strain tensor. 
To complete the model, the group prefactor $K_{0} \epsilon^{2 / 3}$ must be calculated for each cell from the resolved flow. This is done by a structure function matching (Lesieur \& Metais 1996; Voelkl, Pullin \& Chan 2000; Pullin 2000). Essentially, the second-order velocity structure function $\mathscr{F}_{2}(\boldsymbol{r})$ when averaged over the surface of a sphere of radius $\Delta$ gives

$$
\overline{\mathscr{F}}_{2}(\Delta)=\frac{4}{\Delta} \int_{0}^{\pi} E(s / \Delta)\left(1-\frac{\sin s}{s}\right) \mathrm{d} s .
$$

The spectra, (3.12), and the assumption that the exponential can be ignored when evaluating at the separation scale $\Delta$ give the group prefactor as

$$
K_{0} \epsilon^{2 / 3}=\frac{\overline{\mathscr{F}_{2}}(\Delta)}{\Delta^{2 / 3} \mathrm{~A}}
$$

where $\mathrm{A}=4 \int_{0}^{\pi} s^{-5 / 3}(1-\sin s / s) \mathrm{d} s \approx 1.90695$. In practice, $\Delta$ and $\Delta_{c}$ are taken to be the grid spacing, $\Delta x$, and the spherical average of the structure function is computed as a local estimate using a six-point stencil on the resolved scales

$$
\overline{\mathscr{F}}_{2}(\Delta)=\frac{1}{6} \sum_{j=1}^{3}\left(\widetilde{\delta u_{1}^{+}}+\delta{\widetilde{u_{2}^{+}}}^{2}+\delta{\widetilde{u_{3}^{+}}}^{2}+\delta{\widetilde{u_{1}^{-}}}^{2}+\delta{\widetilde{u_{2}^{-}}}^{2}+\delta{\widetilde{u_{3}^{-}}}^{2}\right)_{j},
$$

where $\delta \tilde{u_{i}^{ \pm}}=\tilde{u_{i}}\left(\boldsymbol{x}_{o} \pm \boldsymbol{e}_{j} \Delta\right)-\tilde{u}_{i}\left(\boldsymbol{x}_{o}\right)$ denotes the $i$ th velocity component difference in the unitary direction $\boldsymbol{e}_{j}$ at the point $\boldsymbol{x}_{o}$.

This subgrid model is based on subgrid elements in the form of spiral vortices that are local approximate solutions of the Navier-Stokes equations (Lundgren 1982), and the scalar transport equations (Pullin \& Lundgren 2001) for a constant-density fluid. It has been applied to two-fluid mixing in Rayleigh-Taylor instability (Mattner, Pullin \& Dimotakis 2004) where the process of subgrid mixing of a variable-density fluid is modelled, in the sense of (3.11), via an SGS temperature flux treated as a passive scalar. There is, therefore, no explicit model representation of subgrid baroclinic vorticity production. Insofar as the SGS vorticity spectrum is eschewed in favour of the SGS energy spectrum, this deficiency may not be fatal. The effect of small-scale two-fluid mixing on the velocity spectrum at large wavenumbers and large Reynolds numbers, remains an open question.

\section{Computational approach}

\subsection{Numerical method}

An improved version of the TCD-WENO hybrid method (Hill \& Pullin 2004), based on tuned centre-difference (TCD) stencils is used to integrate the equations of motion in conjunction with a third-order strong-stability preserving (SSP) Runge-Kutta timestepping scheme (Gottlieb, Shu \& Tadmor 2001). The spatial discretization has been constructed explicitly to be shock-capturing, to enforce weak convergence (prediction of the correct shock speeds), and to revert smoothly to a centred stencil with low numerical dissipation and good wave-dispersion properties in turbulent-flow regions away from shocks. Here, the hybrid method uses a bandwidth optimized five-point centre-difference stencil tuned for better modified wavenumber behaviour at the price of a reduction in the order of accuracy from fourth-order to second-order. This tuning was achieved by minimizing the spatial truncation error (Ghosal 1996, 1999) for the Navier-Stokes equations under model assumptions of a von Kármán spectra. In Hill \& Pullin (2004), the TCD scheme was shown to work well on $32^{3}$ LES of 
decaying compressible turbulence with a turbulent Mach number of $M_{t}=0.488$ and an initial Taylor Reynolds number $R e_{\lambda}=175$. Only in thin regions containing shocks does the hybrid method switch to a finite-difference WENO scheme (Jiang \& Shu 1996) whose optimal candidate stencil has been modified to match that of the TCD. The implementation of the hybrid flux-based TCD portion of the scheme used in this paper is the extended version presented in Pantano et al. (2005) which incorporates a flux-based representation for adaptive mesh refinement.

The present flux-based numerical method ensured discrete mass, momentum and energy conservation with unique interpolated flux values at the grid cell wall, even when the scheme switches method. To illustrate the technique, consider a uniform one-dimensional discretization of a function $f(x)$. The fluxes, $F_{i+1 / 2}$ at the right-hand sidewall of the $i$ th computational grid cell, and the numerical approximation of the derivative of $f(x)$ are related through

$$
\left.\frac{\partial f}{\partial x}\right|_{i} \simeq \mathscr{D}_{x} f=\frac{F_{i+1 / 2}-F_{i-1 / 2}}{\Delta x}
$$

where $\Delta x$ denotes the grid spacing and $\mathscr{D}_{x}$, the finite-difference stencil operator. The smallest five-point centred stencil operator has the form

$$
\mathscr{D}_{x} f=\frac{1}{\Delta x}\left(\alpha\left(f_{i+2}-f_{i-2}\right)+\beta\left(f_{i+1}-f_{i-1}\right)\right),
$$

where $\beta=1 / 2-2 \alpha$ is required for at least second-order accuracy; $\alpha=-1 /(12)$ is the standard fourth-order stencil, and $\alpha=-0.197$ corresponds to the TCD stencil. The divergence-like TCD flux is given by

$$
F_{i+1 / 2}=\alpha\left(f_{i+2}+f_{i+1}\right)+(\alpha+\beta)\left(f_{i+1}+f_{i}\right) .
$$

This can be verified by introducing (4.3) into (4.1). To ensure numerical stability, the discrete version of the convective term of the momentum, scalar transport and energy equations are written in the compressible extension of the skew-symmetric form (Blaisdell 1991), such that the summation by parts property is satisfied. This implies that derivatives of quadratic products of two functions $f$ and $g$ are actually evaluated as

$$
\frac{\partial(f g)}{\partial x} \simeq \frac{1}{2}\left(\mathscr{D}_{x}(f g)+f \mathscr{D}_{x} g+g \mathscr{D}_{x} f\right) .
$$

Moreover, it is possible to show that this skew-symmetric form can also be written in flux form (Ducros et al. 2000). For the case of the TCD scheme, the skew-symmetric flux is given by

$$
F_{i+1 / 2}^{T C D}=\frac{1}{2}\left\{\alpha\left[\left(g_{i+1}+g_{i-1}\right)\left(f_{i+1}+f_{i-1}\right)+\left(g_{i+2}+g_{i}\right)\left(f_{i+2}+f_{i}\right)\right]+\beta\left[\left(f_{i+1}+f_{i}\right)\left(g_{i+1}+g_{i}\right)\right]\right\} .
$$

This form is used for the convective terms in the momentum, scalar and energy equation (cast in the internal energy variance conserving form suggested by Honein \& Moin 2004), that reads

$$
\begin{aligned}
& \frac{\partial\left(\bar{\rho} \tilde{u}_{i} \tilde{u}_{j}\right)}{\partial x_{j}} \mapsto \frac{1}{2} \frac{\partial\left(\bar{\rho} \tilde{u}_{i} \tilde{u}_{j}\right)}{\partial x_{j}}+\frac{\bar{\rho} \tilde{u}_{j}}{2} \frac{\partial\left(\tilde{u}_{i}\right)}{\partial x_{j}}+\frac{\tilde{u}_{i}}{2} \frac{\partial\left(\bar{\rho} \tilde{u}_{j}\right)}{\partial x_{j}}, \\
& \frac{\partial\left(\bar{\rho} \tilde{\psi} \tilde{u}_{j}\right)}{\partial x_{j}} \mapsto \frac{1}{2} \frac{\partial\left(\bar{\rho} \tilde{\psi} \tilde{u}_{j}\right)}{\partial x_{j}}+\frac{\bar{\rho} \tilde{u}_{j}}{2} \frac{\partial(\tilde{\psi})}{\partial x_{j}}+\frac{\tilde{\psi}}{2} \frac{\partial\left(\bar{\rho} \tilde{u}_{j}\right)}{\partial x_{j}}
\end{aligned}
$$




\begin{tabular}{lcccc}
\hline & & & & \\
& $\mathrm{II}^{b}$ & $\mathrm{VI}^{b}$ & $\mathrm{VI}^{e}$ & $\mathrm{VII}^{b}$ \\
Incident Mach number & 1.24 & 1.50 & 1.50 & 1.98 \\
Computational grid & $616 \times 128^{2}$ & $388 \times 128^{2}$ & $776 \times 256^{2}$ & $327 \times 128^{2}$ \\
Computational resolution: $\Delta x(\mathrm{~cm})$ & 0.21 & 0.21 & 0.105 & 0.21 \\
Simulation time (ms) & 16.62 & 6.35 & 12.0 & 2.57 \\
CPU hours & 3982 & 972 & 38400 & 544
\end{tabular}

TABLE 3. The computational cost in CPU hours for each of the runs. Simulation $\mathrm{VI}^{e}$ is a higher resolution version of $\mathrm{VI}^{b}$ computed to about twice the experimental time.

$$
\begin{aligned}
\frac{\partial\left((\bar{E}+\bar{p}) \tilde{u}_{j}\right)}{\partial x_{j}} \mapsto & \frac{1}{2} \frac{\partial\left(\bar{\rho} \tilde{e} \tilde{u}_{j}\right)}{\partial x_{j}}+\frac{\bar{\rho} \tilde{u}_{j}}{2} \frac{\partial \tilde{e}}{\partial x_{j}}+\frac{\tilde{e}}{2} \frac{\partial\left(\bar{\rho} \tilde{u}_{j}\right)}{\partial x_{j}}, \\
& +\frac{\tilde{u}_{i}}{2} \frac{\partial\left(\bar{\rho} \tilde{u}_{i} \tilde{u}_{j}\right)}{\partial x_{j}}+\frac{\bar{\rho} \tilde{u}_{i} \tilde{u}_{j}}{2} \frac{\partial \tilde{u}_{i}}{\partial x_{j}}+\bar{p} \frac{\partial u_{j}}{\partial x_{j}}+\tilde{u}_{j} \frac{\partial \bar{p}}{\partial x_{j}},
\end{aligned}
$$

where $\tilde{e}=\bar{E} / \bar{\rho}-\tilde{u}_{k} \tilde{u}_{k} / 2$ is the internal energy.

Around shocks, the WENO scheme naturally computes cell wall fluxes, $F_{i+1 / 2}^{\text {WENO }}$, based on a convex weighting of candidate stencils in an attempt to minimize interpolation across discontinuities. The present hybrid scheme simply selects the WENO fluxes for cells in a tight area around shocks, but uses the centred stencils elsewhere according to the following pressure and density relative curvature criteria

$$
\mathscr{C}=\left\{\boldsymbol{x} \in \mathbf{R}^{3}:\left|\alpha_{p}\right|>c \Delta x^{2},\left|\alpha_{\rho}\right|>c \Delta x^{2}, \alpha_{p} \alpha_{\rho}>0\right\},
$$

where

$$
\begin{gathered}
\alpha_{p}=\frac{\bar{p}_{i+1}-2 \bar{p}_{i}+\bar{p}_{i-1}}{\bar{p}_{i+1}+2 \bar{p}_{i}+\bar{p}_{i-1}}, \\
\alpha_{\rho}=\frac{\bar{\rho}_{i+1}-2 \bar{\rho}_{i}+\bar{\rho}_{i-1}}{\bar{\rho}_{i+1}+2 \bar{\rho}_{i}+\bar{\rho}_{i-1}} .
\end{gathered}
$$

Moreover, all grid cells in a neighbourhood of radius $n \Delta x$ of the cells that satisfy the test condition are marked as containing the discontinuity. The three-dimensional version of this test is used in the simulations. The values of $c$ and $n$ that proved to give the best results were $2.5 \times 10^{3}$ and 5 , respectively. Then, the hybrid flux takes the form

$$
F_{i+1 / 2}= \begin{cases}F_{i+1 / 2}^{W E N O} & \text { in } \mathscr{C}, \\ F_{i+1 / 2}^{T C D} & \text { in } \overline{\mathscr{C}},\end{cases}
$$

where $\overline{\mathscr{C}}$ denotes the complement of $\mathscr{C}$.

\subsection{Simulations}

Four large-eddy simulations were performed and the physical parameters chosen to match the experiments as summarized in table 1. Three of these were at a resolution defined by the grid spacing of $\Delta x=0.21 \mathrm{~cm}$. They ran to the end of the experimental time with modest computational cost (table 3). These simulations were of sufficient resolution to form the basis of our comparisons of mixing-layer width with the experiments. The fourth simulation was of the Mach 1.50 case $\left(\mathrm{VI}^{e}\right)$, it used twice the resolution (i.e. $\Delta x=0.105 \mathrm{~cm}$ ) and was integrated for twice the experimental time to allow for a much more detailed analysis of the flow evolution and the subsequent 
(a)

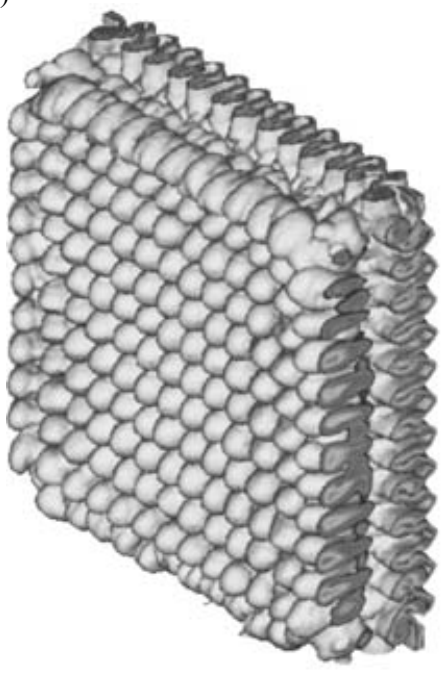

(b)

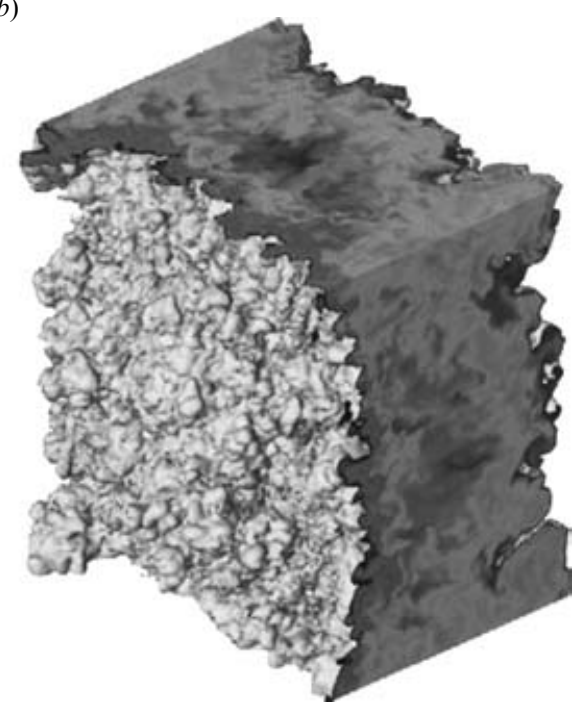

FIgURE 3. Images of the mixing zone for case $\mathrm{VI}^{e}$ at $(a) t=4.8 \mathrm{~ms},(b) t=10 \mathrm{~ms}$.

turbulent decay of the mixing region. This case was chosen in part because of its modest domain size $(L=0.62 \mathrm{~m})$ and the fact that it was the most throughly studied experiment in Vetter \& Sturtevant (1995). Figure 3 shows instantaneous rendering of isosurfaces of a mixture fraction at several times during the evolution of case Mach 1.50, exemplifying the different states of the flow.

The simulations were all performed by a parallel FORTRAN90 program on QSC, an unclassified Tru64 cluster at the Los Alamos National Laboratory; each processor is an Alpha EV6 with 4GB of memory and a clock speed of $1.25 \mathrm{GHz}$.

\section{Simulation results (traditional statistics)}

Traditional statistical results (i.e. those of the resolved fields) obtained from the LES are summarized here. First, the mixing-layer width is defined and comparisons with the experiments are made based on the modest resolution runs. The higher-resolution Mach 1.50 mixing-layer width is also obtained up to times that are much longer than the experimental time of $6.35 \mathrm{~ms}$ and the turbulent kinetic energy is also computed; this analysis helps to gain a better understanding of the importance of the shocks and subsequent expansion waves that evolve in the domain. Plane-averaged flow quantities on both the resolved scale and subgrid are presented, illustrating the low turbulent Mach number in the flow and the role of the subgrid in dissipation and kinetic energy. Following this, the mixing zone is examined in the late time stages after the shock and expansion interactions. During this time, the mixing zone grows slowly at best and exhibits some of the characteristics of decaying weakly compressible turbulence. Two statistical measurements are introduced: the instantaneous plane average, that for an arbitrary field $f$ is defined as

$$
\langle f(x, t)\rangle=\frac{1}{\mathscr{A}} \iint f(x, y, z, t) \mathrm{d} y \mathrm{~d} z,
$$




$\begin{array}{lccc} & \mathrm{II}^{b} & \mathrm{VI}^{b} & \mathrm{VII}^{b} \\ \text { Incident Mach number } & 1.24 & 1.50 & 1.98 \\ \text { Experimental final layer thickness (cm) } & 13.7 & 10.2 & 8.25 \\ \text { Computed final thickness (cm) } & 12.2 & 10.6 & 8.0 \\ \text { Measurement time (ms) } & 16.50 & 6.25 & 2.50 \\ \text { Simulation time (ms) } & 16.62 & 6.35 & 2.57 \\ \text { First shock time in simulation (ms) } & 0.119 & 0.098 & 0.073\end{array}$

TABLE 4. A comparison of the mixing width at the end of the experiment with the computed mixing width at a similar time in the simulation. The experimental times and widths are approximate as they were measured from figure 6 of Vetter \& Sturtevant (1995).

where $\mathscr{A}$ is the $y / z$ cross-sectional area of the tube $\left(\mathscr{A}=0.27^{2} \mathrm{~m}^{2}\right)$, and the volume average

$$
|f(t)|=\frac{1}{\mathscr{V}} \iiint f(x, y, z, t) \mathrm{d} x \mathrm{~d} y \mathrm{~d} z
$$

where $\mathscr{V}$ is the volume of the computational domain. Note that (5.2) is equivalent to integrating (5.1) in the $x$-direction and dividing by the length of the domain.

Spectra are computed in the centre of the mixing zone to confirm that universal $k^{-5 / 3}$ scaling is recovered. Reynolds numbers and dissipation lengths are calculated from the flow and the bubble and spike mixing process is analysed by examining the resolved variances. Additionally the mixing proprieties across the width of the layer are investigated.

\subsection{Mixing-layer growth and comparison with experiment}

To make contact with the mixing-zone growth rates estimated by Vetter \& Sturtevant (1995) we define a mixing width $\delta_{\mathrm{MZ}}$ from the planar averaged mixture fraction, according to

$$
\delta_{\mathrm{MZ}}(t)=4 \int_{\text {tube }}(1-\langle\psi\rangle)\langle\psi\rangle \mathrm{d} x .
$$

An analytic example can be helpful in understanding this measure better. Assume a simple average profile that represents the transition from air $(\psi=0)$ to $\mathrm{SF}_{6}(\psi=1)$ and centred at $x_{c}$ in an infinitely long tube to be of the form $\langle\psi\rangle=\left(1+\tanh \left(2\left(x-x_{c}\right) / h\right)\right) / 2$. Introducing this function into (5.3) gives the expected mixing width $\delta_{\mathrm{MZ}}=h$. In the actual experiments, measurements were taken from photographs of the mixing zone, but the way in which these measurements of the width were made was not reported. This uncertainty tempers the degree to which we draw conclusions in our comparison, and, more broadly, affects the comparison of most turbulent RMI experiments to theoretical results and computational simulations.

The computed widths of the mixing zone for the different modest-resolution simulations are shown in figure 4, where the experimental growth rates are plotted as solid lines of duration corresponding to the experimental observation times. The agreement in growth rate for all three simulations are quite reasonable. As the simulation initial perturbations were much larger than those inferred for the experiment, no actual agreement in the mixing-zone width was anticipated in the early stages of the simulation. The measured width during the times prior to reshock were about $50 \%$ of the computed widths. The post-reshock thicknesses agree well with the experiment, table 4, with discrepancy of the order of $4 \%$ for the Mach 1.50 and 1.98 cases and $10 \%$ for the Mach 1.24 case. Note that $t=0$ in the experiments corresponds 


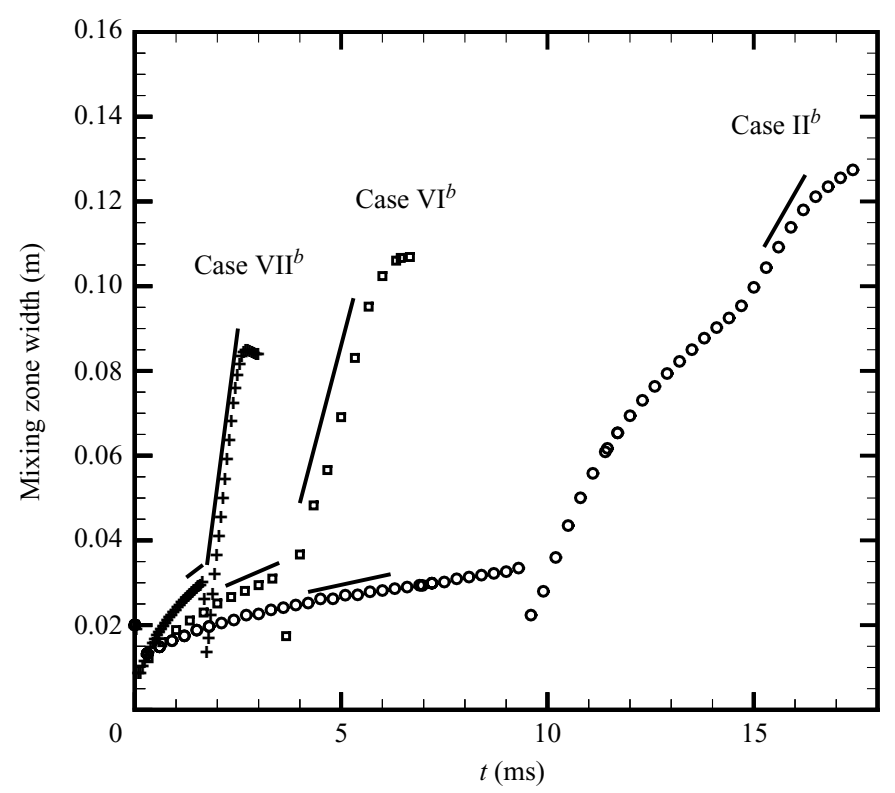

FIgURE 4. The evolution of the mixing-layer width $\delta_{\mathrm{MZ}}$. The growth rates inferred from the experiments are indicated as straight lines with the appropriate slopes drawn in the approximate times over which they were observed. See table 4 for Mach numbers.

to the first shock interaction, but in simulations, the shock is initialized at $5 \mathrm{~cm}$ to the left of the interface; hence, the offset in measurement times. The much better agreement after reshock is consistent with the detailed Mach 1.50 experiment of Vetter $\&$ Sturtevant (1995) (summarized in their figure 8) in which the initial conditions were varied by altering the membrane and wire mesh configuration. In the case of two different initial amplitudes, they found very similar post-reshock behaviour and nearly identical widths were recorded.

Figure 5 shows the computed mixing width for the higher resolution Mach 1.50 run where the experimentally measured growth rates of Vetter \& Sturtevant (1995) are also indicated. Both shock-interface interactions are apparent in the form of sharp compressions of the mixing zone. These compressions are followed by periods of mixing-zone growth. The interaction of an expansion with the growing mixing layer can also be observed in the slight change of the layer growth rate at $t \approx 4.7 \mathrm{~ms}$. This expansion is generated by the reshock event, after it reflects from the wall, and impinges on the mixing layer (see figure 2). The evolution of the mixing width after reshock can then be divided into distinct parts: the initial growth from the reshock which starts to decay around $4.5 \mathrm{~ms}$, further growth stimulated by the reflectedexpansion event, and peaking at $6 \mathrm{~ms}$ followed finally by turbulent saturation and a subsequent very slow period of growth. These stages in the life of the post-reshock turbulent mixing zone can be seen clearly in the turbulent kinetic energy of the flow.

\subsection{Turbulence statistics}

Planar-averaged statistics of the different flow fields for the case with initial shock Mach number of 1.50 are reported here. Results are presented at two times: $t=1 \mathrm{~ms}$ shortly after the initial shock interaction while the mixing layer is initially growing but not fully turbulent, and $t=10 \mathrm{~ms}$ after the reshock and expansion wave events at a time when the mixing zone is fully turbulent. The Favre-plane-averaged turbulence 


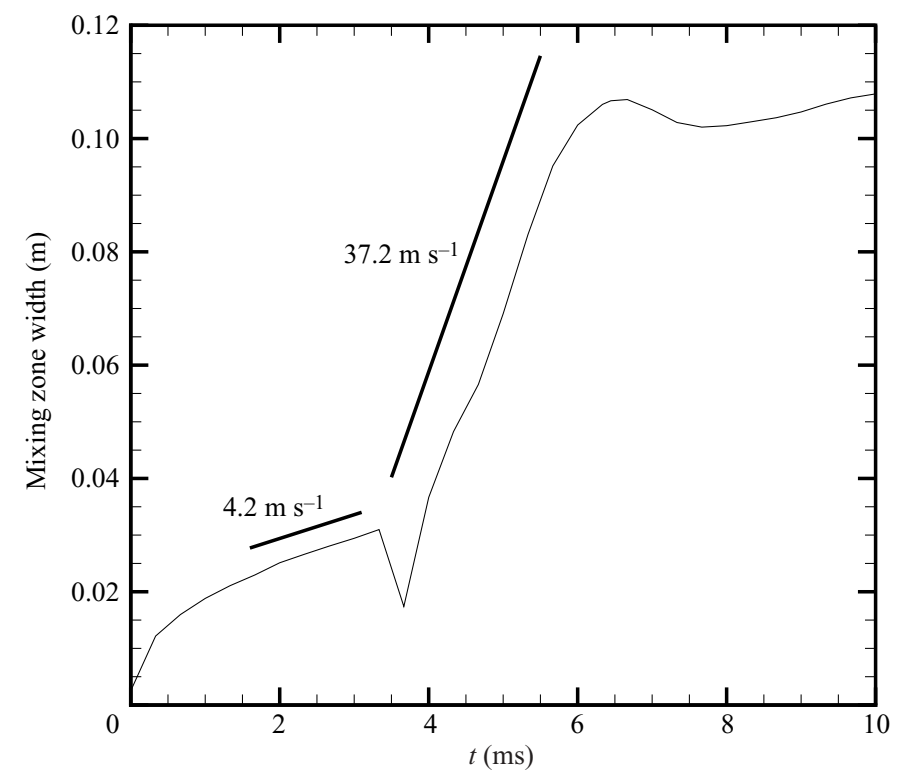

Figure 5. The evolution of the mixing-layer width $\delta_{\mathrm{MZ}}$. The experimentally measured values are indicated as are the approximate times in which they were observed.

statistics of interest include the resolved-scale turbulent kinetic energy

$$
\langle\tilde{K}\rangle=\frac{1}{2}\left(\frac{\left\langle\bar{\rho} \tilde{u}_{k} \tilde{u}_{k}\right\rangle}{\langle\bar{\rho}\rangle}-\frac{\left\langle\bar{\rho} \tilde{u}_{k}\right\rangle\left\langle\bar{\rho} \tilde{u}_{k}\right\rangle}{\langle\bar{\rho}\rangle^{2}}\right),
$$

the resolved-scale turbulent dissipation

$$
\left\langle\epsilon_{r e s}\right\rangle=\frac{\left\langle d_{i j}^{\prime} \tilde{S}_{i j}^{\prime}\right\rangle}{\langle\bar{\rho}\rangle}
$$

the subgrid turbulent kinetic energy

$$
\langle\tilde{k}\rangle=\frac{\left\langle\tau_{k k}\right\rangle}{2\langle\bar{\rho}\rangle},
$$

and the subgrid energy transfer

$$
\left\langle\epsilon_{s g s}\right\rangle=-\frac{\left\langle\tau_{i j}^{\prime} \tilde{S}_{i j}^{\prime}\right\rangle}{\langle\bar{\rho}\rangle},
$$

where primes denote fluctuations with respect to the plane-average. The total turbulent kinetic energy is estimated then as $K=\langle\tilde{K}\rangle+\langle\tilde{k}\rangle$ and the turbulent dissipation as $\epsilon=\left\langle\epsilon_{\text {sgs }}\right\rangle+\left\langle\epsilon_{\text {res }}\right\rangle$. These quantities are used to define the turbulent intensity,

$$
u^{\prime}=\sqrt{\frac{2 K}{3}}
$$

and the turbulent Mach number

$$
M_{t}=\frac{u^{\prime}}{\langle c\rangle}
$$



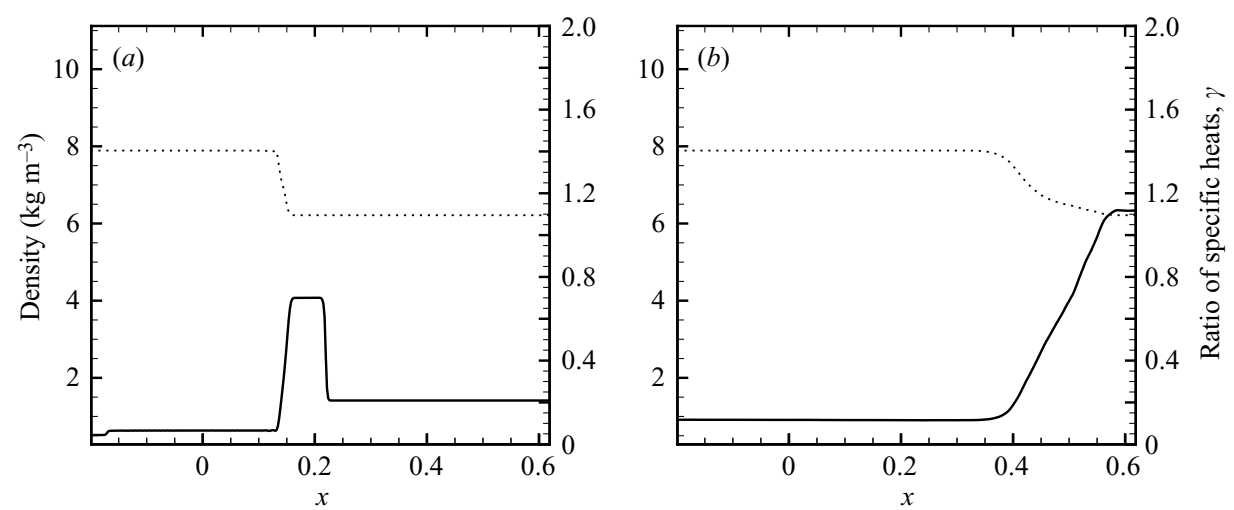

Figure 6 . The $(y, z)$-plane averaged density $\langle\bar{\rho}\rangle$ (solid line) and ratio of specific heats $\langle\tilde{\gamma}\rangle$ (dotted line) profiles $(a) t=1 \mathrm{~ms}$, during the evolution of the flow. $(b) t=10 \mathrm{~ms}$.

using the average of the speed of sound $c=\sqrt{\tilde{\gamma} \bar{p} / \bar{\rho}}$. A turbulence integral scale can also be defined as

$$
\ell=\frac{u^{\prime 3}}{\epsilon}
$$

allowing the calculation of the turbulent Reynolds number as

$$
R e_{T}=\frac{u^{\prime} \ell}{\langle v\rangle} .
$$

In figure 6 , the density at time $t=1 \mathrm{~ms}$ shows a small reflected shock near $x=-0.2 \mathrm{~m}$ travelling to the left. The contact, which will evolve into the mixing zone, can be seen in both the density and the ratio of specific heats near $x=0.15 \mathrm{~m}$ following behind the transmitted shock $(x=0.225 \mathrm{~m})$ as both travel to the right. In the later time plot, $t=10 \mathrm{~ms}$, the shock has reflected off the closed end of the tube at $x=0.62 \mathrm{~m}$, reshocked the mixing zone and left the domain. Here, the much larger extent of the mixing zone is clearly visible. The shocks and expansion interactions with the mixing zone are inherently compressible effects and form the principal mechanisms for the deposition of vorticity in the mixing zone.

One useful feature of the stretched vortex subgrid model is its ability to estimate directly local subgrid quantities such as the local subgrid kinetic energy $\tilde{k}$ and the energy transfer off-grid. Figures 7 and 8 show that $\langle\tilde{k}\rangle$ is of the order of 10 to $20 \%$ of the resolved counterpart and, as expected from a proper LES, the planaraveraged subgrid energy transfer, $\left\langle\epsilon_{s g s}\right\rangle$ is about 10 times the resolved dissipation prior to reshock and 100 times larger after reshock. This is another indication that the turbulence scales, down to the unresolvable range, fully develop only after reshock.

Figure 9 shows profiles of turbulent Mach number at two different times. It is observed that the relative effects of compressibility during the evolution of the mixing zone peak at its centre. While the plot at $t=1 \mathrm{~ms}$ has a much lower overall turbulent Mach number, the larger peak of $M_{t}=0.07$ in the post-reshock, $t=10 \mathrm{~ms}$, stage is clearly in only the weakly compressible range. Thus, excluding the shock interaction events, compressibility effects in the turbulence are not large for this simulation.

The length scale $\ell$ and turbulent Reynolds number $R e_{T}$ are presented in a time range $7 \mathrm{~ms}-10 \mathrm{~ms}$ which starts shortly before the energy cascade forms, as indicated by the development of the $k^{-5 / 3}$ scaling by time $t=7.6 \mathrm{~ms}$, shown next. In figure 10 , it can be seen that the length scale and Reynolds number decay during this period at the centre of the mixing zone as the large bubbles created by reshock interact and 

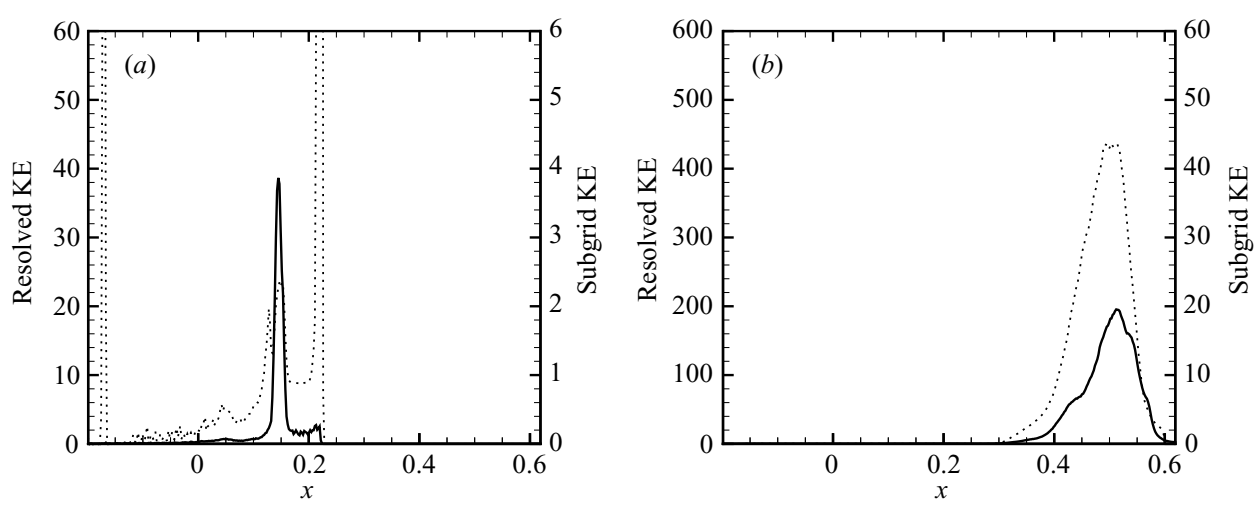

FiguRE 7. The $(y, z)$-plane averaged resolved scale turbulent kinetic energy (solid line) compared with the subgrid kinetic energy as estimated by the model (dotted line). Note the order of magnitude difference in the scale of the plots. All quantities in MKS units. $(a) t=1 \mathrm{~ms}$, (b) $t=10 \mathrm{~ms}$.
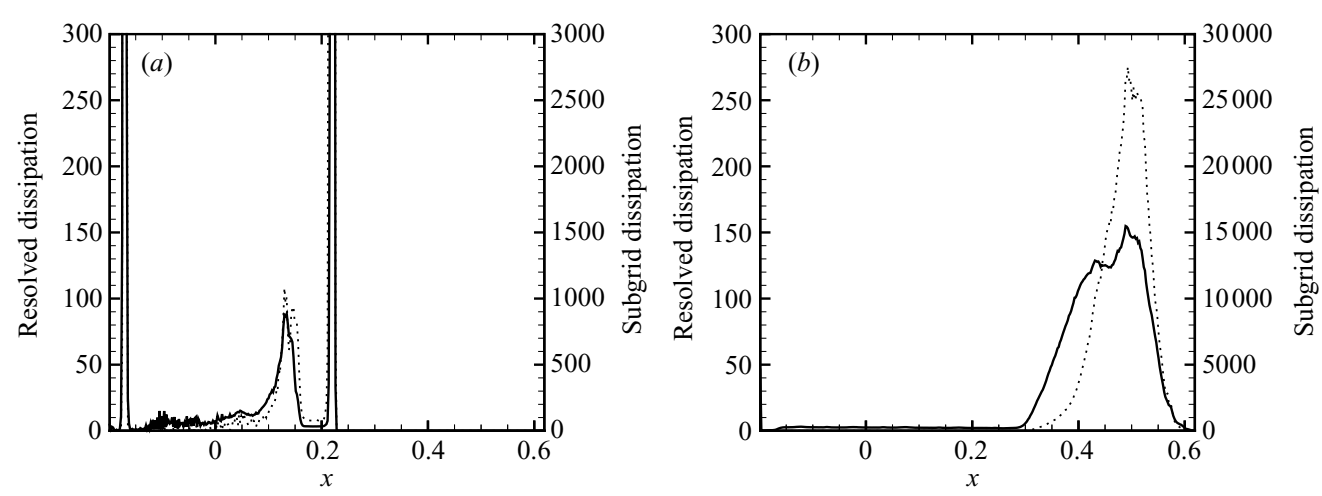

FIGURE 8 . The $(y, z)$-plane averaged resolved scale dissipation rate (solid line) compared with the subgrid dissipation as estimated by the model (dotted line). All quantities in MKS units. (a) $t=1 \mathrm{~ms},(b) t=10 \mathrm{~ms}$.
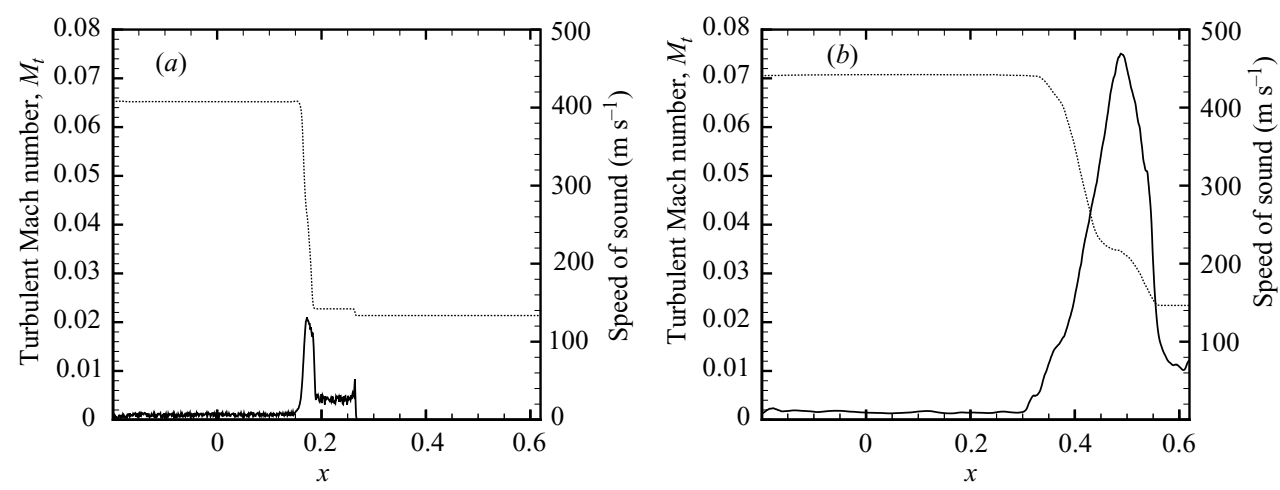

FIGURE 9. The $(y, z)$-plane averaged resolved scale turbulent Mach number (solid line) compared with plane averaged local speed of sound (dotted line). (a) $t=1 \mathrm{~ms},(b) t=10 \mathrm{~ms}$.

compete. It is noted that $\ell$ is comparable to the $2 \mathrm{~cm}$ spacing in the initial interface perturbation and although $\langle\tilde{K}\rangle$ has dropped substantially by $t=7 \mathrm{~ms}, R e_{T}$ is still in the range of 30000 to 100000 . 

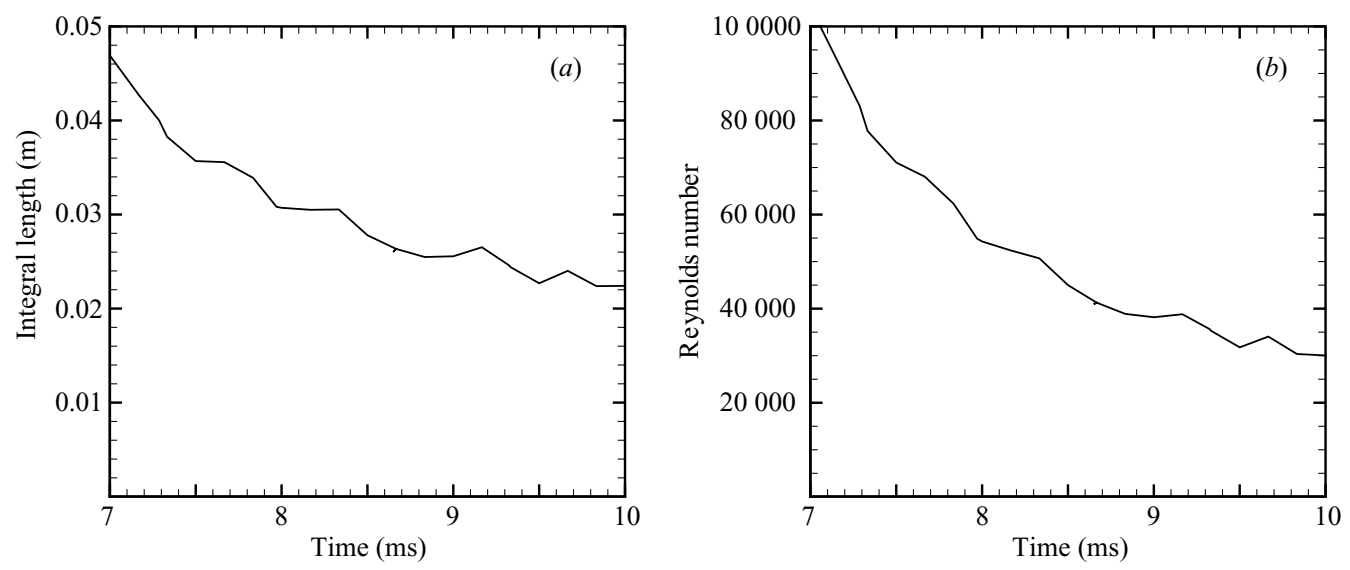

FIGURE 10. ( $a$ ) The plane-averaged integral length scale, $l$, and $(b)$ turbulent Reynolds number, $R e_{T}$, computed in the centre of the mixing zone as a function of time.

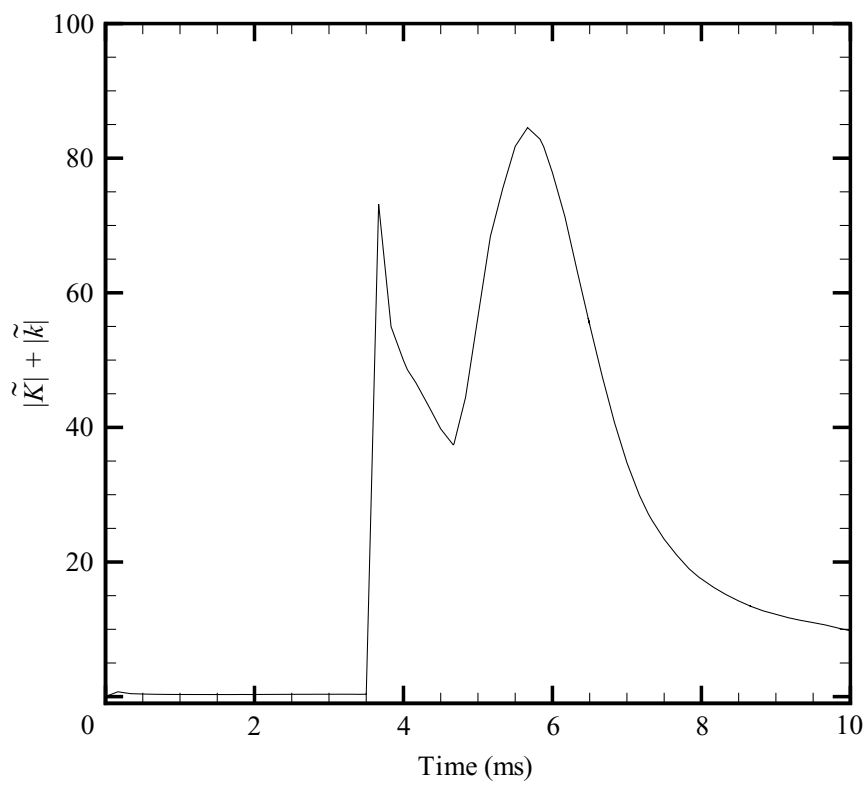

FiguRE 11. Volume-averaged total turbulent kinetic energy $|\tilde{K}|+|\tilde{k}|$ as a function of time for the $M=1.50$ case.

Finally, a global turbulence measure can be obtained from the volume-averaged turbulent kinetic energy. The total turbulent kinetic energy deposited by the shockcontact interactions as well as expansion fan-contact interactions can be measured using this statistical quantity. Figure 11 shows the total amount of energy deposited by the initial shock, visible as a very small bump close to the time origin, as well as that owing to the reshocking event, at $3.5 \mathrm{~ms}$. Following a steep decay in energy forming the first stage in the post-reshock mixing zone's life, a subsequent interaction with the expansion fan, shown in the wave diagram (figure 2), deposits a relatively large amount of energy over the duration of approximately $1 \mathrm{~ms}$, peaking near $6 \mathrm{~ms}$. This last vorticity deposition corresponds to the second period of post-reshock growth. After these events, there is a very slow period of decay since none of the additional 


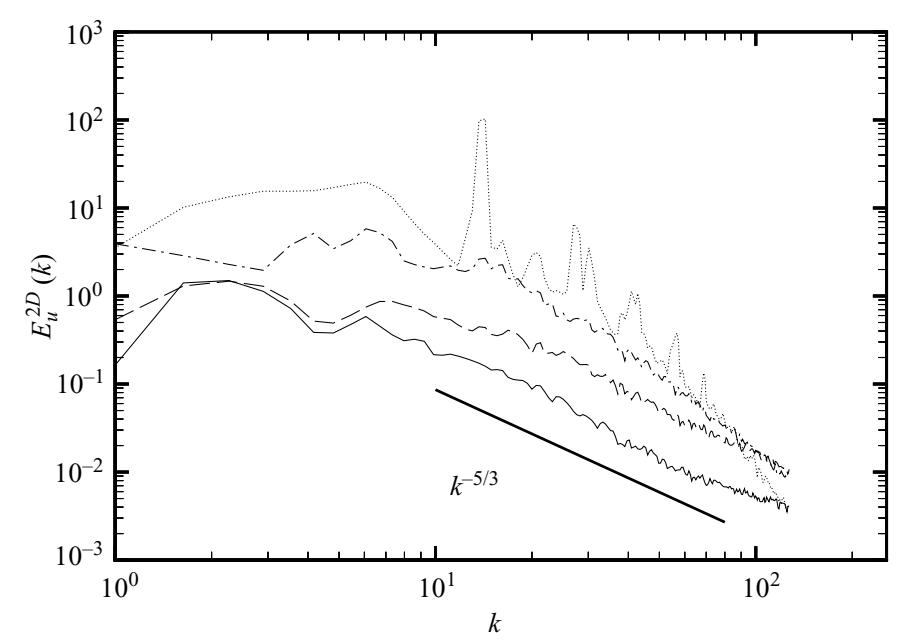

FIGURE 12. Radial power spectra of velocity $E_{u}^{2 D}(k)$ computed in the centre plane of the TMZ at four different times: $t=4.5 \mathrm{~ms}$ (dotted line), $t=6.5 \mathrm{~ms}$ (dashed-dot line), $t=7.6 \mathrm{~ms}$ (dashed line) and $t=10 \mathrm{~ms}$ (solid line). All computed wavenumbers shown and $k_{\max }=128$.

weak expansion fans and compression waves coming from the wall posses a sufficiently large pressure gradient to deposit noticeable amounts of vorticity. It is notable that the first expansion fan deposits substantial amounts of vorticity, and kinetic energy. This energy is comparable to that of the re-shocking event and occurs because the expansion-fan interaction takes place over a longer time period during which the mixing zone is rather thick, with a wide range of spatial scales and gradients in the density field.

\subsection{Velocity, density and scalar spectra}

The simulation domain was designed with periodic boundary conditions in the crosssection of the tube, in part to allow for two isotropic directions in the flow and to enable the calculation of instantaneous radial spectra. The radial spectra of an arbitrary function $f(y, z)$ is defined as

$$
E_{f}^{2 D}(k)=\frac{1}{2} k \int_{0}^{2 \pi}\left|\hat{F}\left(k, \theta_{k}\right)\right|^{2} \mathrm{~d} \theta_{k}
$$

where $\hat{F}$ is the Fourier transform of $f$ in polar wavenumber space with $k$ and $\theta_{k}$ denoting the radial and azimuthal wavenumbers. In practice, this is most easily calculated in Cartesian wavenumber space $k_{y}, k_{z}$ by annular bin-counting. The spectra of the individual velocity components $u=u_{1}, v=u_{2}, w=u_{3}$ as well as of the density $\rho$ and scalar $\psi$ was calculated using (5.12).

The radial velocity spectra was always calculated at the plane located in the centre of the mixing zone. The results shown in figure 12 indicate that the spectra assumes a persistent $k^{-5 / 3}$ scaling after the passage of the expansion fan and a reorganization of the deposited kinetic energy. The expansion interaction starts at about $4.7 \mathrm{~ms}$ and continues until $6 \mathrm{~ms}$, as seen in the wave diagram figure 2 and in the plot of the total turbulent kinetic energy figure 11 . The Kolmogorov-like $k^{-5 / 3}$ energy spectra develops by $t=7.6 \mathrm{~ms}$ and persists for the remainder of the simulation. Similarly, the density and scalar spectra also develop a persistent $k^{-5 / 3}$ scaling by this time. 


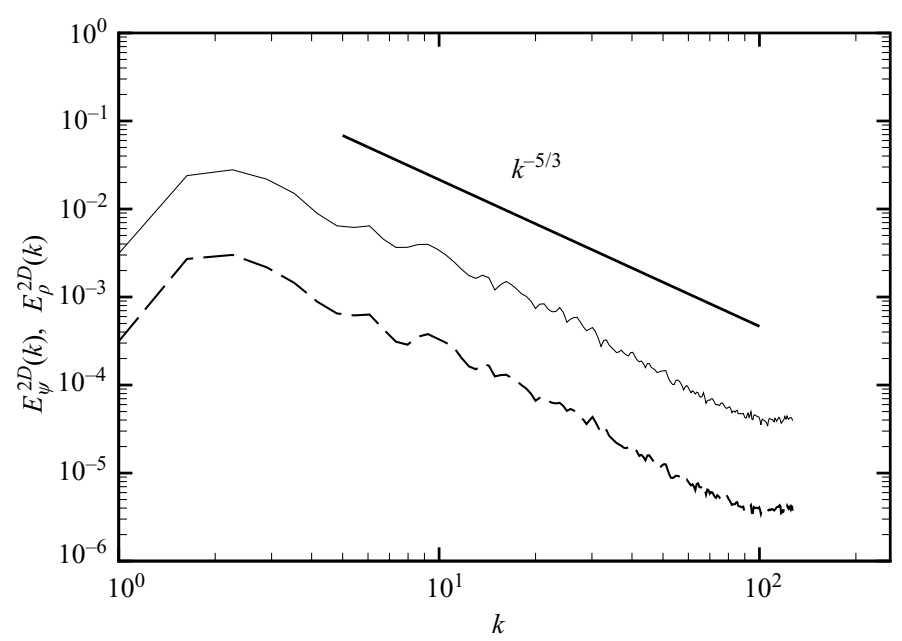

FIGURE 13. Radial power spectra of density (solid line) and mixture fraction (broken line), i.e. $E_{\rho}^{2 D}(k)$ and $E_{\psi}^{2 D}(k)$, at $t=10 \mathrm{~ms}$ computed in the centre plane of the TMZ. All computed wavenumbers shown: $k_{\max }=128$.
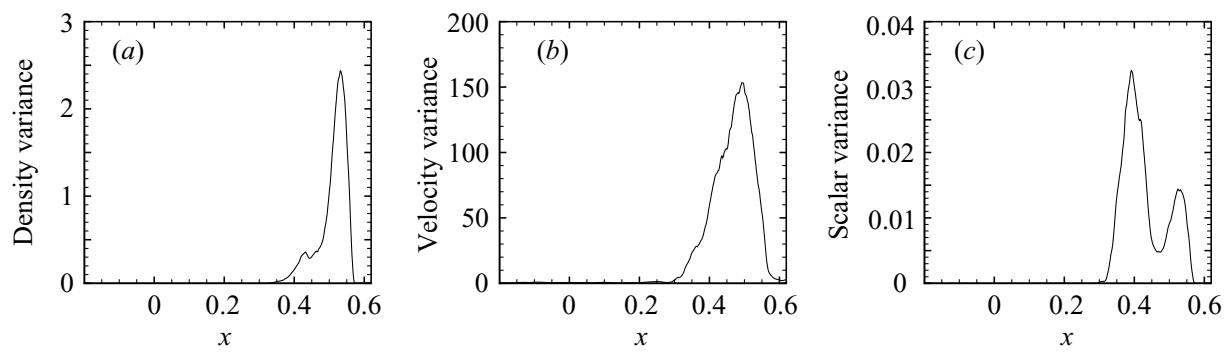

FIGURE 14. The $x$-plane variance of the resolved scale density, mixture fraction, and velocity at $t=10 \mathrm{~ms}$. (a) $\left\langle\bar{\rho}^{2}\right\rangle-\langle\bar{\rho}\rangle^{2},(b)\left\langle\bar{\rho} \bar{u}_{i}^{2}\right\rangle /\langle\bar{\rho}\rangle-\left\langle\bar{\rho} \bar{u}_{i}\right\rangle^{2} /\langle\bar{\rho}\rangle^{2},(c)\left\langle\bar{\rho} \bar{\psi}^{2}\right\rangle /\langle\bar{\rho}\rangle-\langle\bar{\rho} \bar{\psi}\rangle^{2} /\langle\bar{\rho}\rangle^{2}$.

Figure 13 demonstrates that while scalar and density are not uniquely related $(\psi$ is constrained mathematically to be $0 \leqslant \psi \leqslant 1$ and obeys a different governing equation) their spectra correlate well. The highest resolved wavenumbers in figure 13 show minor effect of aliasing errors. We emphasize that no explicit filtering of any kind was performed in the present LES and WENO is not used in this region of the flow.

\subsection{Mixing statistics}

The mixture fraction field $\psi$ parameterizes the degree of mixing of the two gases. Although the flow at late times is quite turbulent, mixing statistics display inhomogeneities associated with the non-isotropic direction $x$ at all times during our simulations. Physically, this is related to the presence of different gasses and the very different bubble-spike structures characteristically observed on either side of the mixing zone. We examine the statistics of the mixture fraction in planar cuts taken from the centre of the mixing zone, $x_{c}$, as well as from planes one quarter of the mixing width $\delta_{\mathrm{MZ}}$ from the centre on either side $x_{q}^{ \pm}=x_{c} \pm \delta_{\mathrm{MZ}} / 4$.

The variance for the density, velocity and mixture fraction, figure 14, highlight the action of the large scales in the flow. Although the density variance is clearly stronger on the $\mathrm{SF}_{6}$ side, the highest turbulent velocities are found closer to the centre 
of the mixing zone. The two peaks in the mixture fraction variance correspond to dominantly bubble and dominantly spike portions of the mixing zone separated by a region of low variance. The energetic structures penetrating into air from the mixing zone produce much larger mixture fraction variances.

To provide a more complete picture of the mixing evolution of the two gases, we investigate now the behaviour of the mixture fraction p.d.f. For variable density flows, it is natural to employ the Favre p.d.f. (Bilger 1977), formally obtained from the Reynolds joint density-mixture fraction p.d.f., $\mathrm{P}(\rho, \psi ; \boldsymbol{x}, t)$, through,

$$
\tilde{\mathrm{P}}(\psi ; \boldsymbol{x}, t)=\frac{1}{\langle\rho\rangle} \int \rho \mathrm{P}(\rho, \psi ; \boldsymbol{x}, t) \mathrm{d} \rho,
$$

where the independent variables $\rho$ and $\psi$ in the p.d.f.s denote the sampling variables in this context, not the LES fields. Moreover, we remark that, strictly speaking, $\mathrm{P}$ and $\tilde{\mathrm{P}}$ represent the p.d.f.s of resolved field quantities when obtained from the LES. They do not denote the p.d.f.s of the total fields, which would include the information of the unresolved scales in the LES. We defer the question of the effect of the unresolved scales on the p.d.f. to $\S 7.3$.

The Favre p.d.f.s, $\widetilde{\mathrm{P}}(\psi)$, as a function of time are shown in figure 15 for the three planes in the mixing zone previously specified. These p.d.f.s were formed by constructing the histograms of resolved mixture fraction, $\tilde{\psi}$, from the LES at planes of constant $x$. These results have been obtained at times that correspond, roughly, to instants just before reshock, just after reshock, just past the peak of kinetic energy deposition by the rarefaction-wave interaction and the end of the simulation, respectively. In the initial phase before reshock (figure 15a), little mixing has taken place. This is evident from the fact that the planar $\tilde{\mathrm{P}}(\psi)$ are very intermittent and most of the fluid is composed of unmixed gases. The two large peaks at the extreme values of mixture fraction, 0 and 1 , are evidence of this state of the gas. This is the phase where the inviscid linear and nonlinear instability mechanisms of RMI dominate; physical and subgrid diffusion have not had sufficient time to act. Almost immediately after reshock (figure $15 b$ ), very fast mixing caused by the vorticity deposited in the mixing zone by the shock leads to a $\tilde{\mathrm{P}}(\psi)$ with a strong central mode for the plane at $x_{c}$. The structure of $\tilde{\mathrm{P}}(\psi)$ at the other two planes close to the pure gases have also changed. The two intermittent peaks at the extreme values of mixture fraction have moved towards the centre of the figure and are now less pronounced. Some adjacent broadening of $\tilde{\mathrm{P}}(\psi)$ including the appearance of plateau is also observed. Before the interaction with the rarefaction wave becomes visible (figure $15 c$ ), $\tilde{\mathrm{P}}(\psi)$ at the plane in the centre and that close to the $\mathrm{SF}_{6}$ side become even more unimodal, with peaks that are well correlated with the average value of mixture fraction at that plane. The p.d.f. at the remaining plane, air side, still preserves a strong degree of intermittency with large amounts of unmixed air. Long after the interaction with the rarefaction wave and the subsequent development of the energy cascade (figure $15 d$ ), the most salient features of $\tilde{\mathrm{P}}(\psi)$ have not changed. We do observe that the width, or variance, of $\tilde{\mathrm{P}}(\psi)$ has narrowed somewhat; indicating further mixing progress. A salient feature of the curves for the central plane and that close to the $\mathrm{SF}_{6}$ side is that the enhanced mixing is non-uniform, or rather forced. We observe the development of two small peaks diametrically opposed to the location where we had seen a single peak. Our observation is that the rarefaction wave deposits more energy on those physical regions of the domain where the density gradient is largest. While $\tilde{\mathrm{P}}(\psi)$ itself is a single-point quantity and thus deprived of scale information, it is reasonable to speculate that the largest density gradients will exist in the region between the 

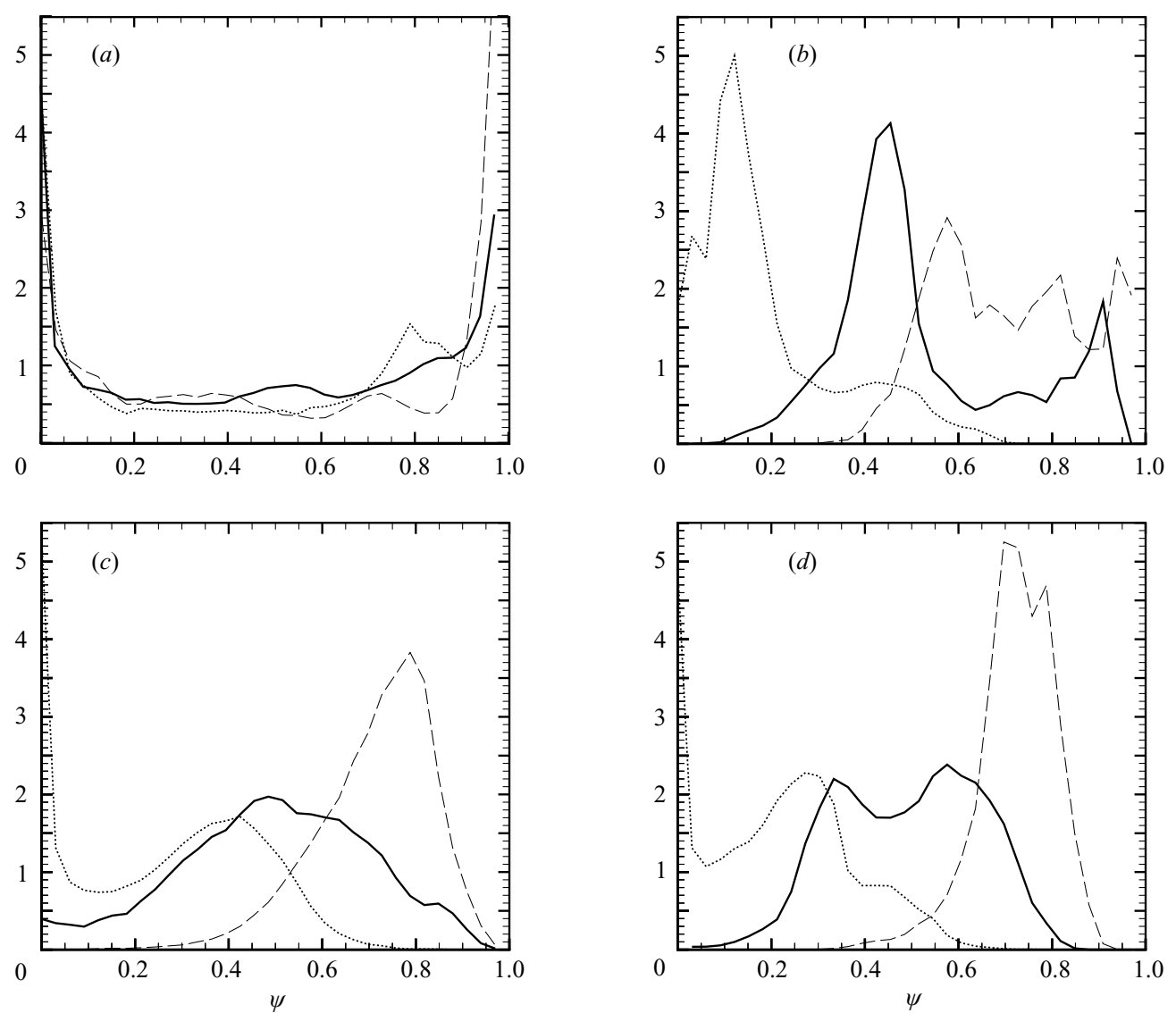

FIGURE 15. Probability density function $\tilde{\mathrm{P}}(\psi)$ of the mixture fraction at different times calculated from the resolved scales only, across three planes of the mixing zone: $x_{c}-0.25 \delta_{\mathrm{Mz}}$ (dotted line), $x_{c}$ (continuous line) and $x_{c}+0.25 \delta_{\mathrm{MZ}}$ (dashed line). (a) $t=3 \mathrm{~ms},(b) 3.6 \mathrm{~ms}$, (c) $7 \mathrm{~ms},(d) 10 \mathrm{~ms}$.

unmixed gasses and the relatively well-mixed core of the TMZ. Then, more vigorous mixing should take place in what appears to be the tails of $\tilde{\mathrm{P}}(\psi)$. This bimodal character of the central $\tilde{\mathrm{P}}(\psi)$ persists as the bubbles and spikes continue to transport partially mixed gases from the outer regions of the mixing zone. We do expect that as time advances further, the bimodal character of $\tilde{\mathrm{P}}(\psi)$ should evolve into a single mode since the remaining rarefaction waves emanating from the wall are very weak.

\section{Subgrid continuation}

The structural nature of the stretched-vortex subgrid model can be used to develop a multiscale treatment of the subgrid activity. This is done in a way which is fully consistent with its implementation when determining the subgrid fluxes in LES. Velocity spectra, including the anisotropic components, can be estimated directly for scales below the cutoff scale from the formulae previously presented. Subgrid continuation of the scalar spectra requires one additional assumption to estimate the effect of Schmidt number, and subgrid mixing statistics require further assumptions of the form of the subgrid p.d.f. Here we outline the mechanics for calculating these continuations in the 
context of the velocity spectra. In the subsequent sections, the additional modelling assumptions required for the scalar are discussed and we present results.

The two-dimensional velocity spectra may be calculated in terms of $k_{r}$, the radial component of wavenumber vector $\boldsymbol{k}=\left(k_{1}, k_{2}, k_{3}\right)$ in polar coordinates. This is a lengthy calculation which closely follows the detailed derivation of the one-dimensional spectra given by Pullin \& Saffman (1994). For that reason, only the main results are outlined here. We begin with the two-point velocity and vorticity correlation tensors

$$
\begin{aligned}
R_{i j}(\boldsymbol{r}) & =\left\langle\left\langle u_{i}(\boldsymbol{x}) u_{j}(\boldsymbol{x}+\boldsymbol{r})\right\rangle\right\rangle, \\
W_{i j}(\boldsymbol{r}) & =\left\langle\left\langle\omega_{i}(\boldsymbol{x}) \omega_{j}(\boldsymbol{x}+\boldsymbol{r})\right\rangle,\right.
\end{aligned}
$$

where $\langle\langle\cdot\rangle\rangle$ denote ensemble averaging and we omit the tilde notation of the velocity vector since we are dealing with the complete velocity vector. The corresponding Fourier transforms are given by

$$
\begin{aligned}
R_{i j}(\boldsymbol{r}) & =\iiint \Phi_{i j}(\boldsymbol{k}) \exp (\mathrm{i} \boldsymbol{k} \cdot \boldsymbol{r}) \mathrm{d} k_{1} \mathrm{~d} k_{2} \mathrm{~d} k_{3}, \\
W_{i j}(\boldsymbol{r}) & =\iiint \Omega_{i j}(\boldsymbol{k}) \exp (\mathrm{i} \boldsymbol{k} \cdot \boldsymbol{r}) \mathrm{d} k_{1} \mathrm{~d} k_{2} \mathrm{~d} k_{3} .
\end{aligned}
$$

Pullin \& Saffman (1994) have shown that without assumptions of isotropy, the vorticity correlation tensor may also be expressed as

$$
\begin{aligned}
W_{i j}(\boldsymbol{r})= & \frac{1}{2 \mathscr{V}} \sum_{m} l_{m} \int_{-\infty}^{\infty} \int_{-\infty}^{\infty} \int_{0}^{\pi} \int_{0}^{2 \pi} \int_{0}^{2 \pi}\left|\hat{\omega}^{m}\left(\kappa_{1}, \kappa_{2}, t\right)\right|^{2} \\
& \times \exp \left(-\mathrm{i} \kappa_{1} r_{1}^{\prime}-i \kappa_{2} r_{2}^{\prime}\right) U_{3 i} U_{3 j} \mathscr{P}\left(\alpha^{\prime}, \beta^{\prime}, \gamma^{\prime}\right) \mathrm{d} \kappa_{1} \mathrm{~d} \kappa_{2} \sin \alpha^{\prime} \mathrm{d} \alpha^{\prime} \mathrm{d} \beta^{\prime} \mathrm{d} \gamma^{\prime},
\end{aligned}
$$

where the sum is over an ensemble of vortices, length $l_{m}$, whose orientations with respect to the laboratory frame of volume $\mathscr{V}$ are described by the p.d.f., $\mathscr{P}$, and the Euler angles $\alpha^{\prime}, \beta^{\prime}, \gamma^{\prime}$. The matrix $U_{i j}$ is a unitary rotation operator that maps vortex, $r_{j}^{\prime}$, and laboratory coordinates, and $\hat{\omega}^{m}\left(\kappa_{1}, \kappa_{2}\right)$ is the Fourier transform of the vorticity expressed in the frame of the vortex.

An expression for the two-dimensional energy spectrum may be arrived at by first defining the two-dimensional energy tensor as

$$
E_{i j}^{2 D}\left(k_{r}\right)=\frac{k_{r}}{2} \int_{0}^{\infty} \int_{0}^{2 \pi} \Phi_{i j} \mathrm{~d} \theta \mathrm{d} k_{3},
$$

where $k_{1}=k_{r} \cos \theta, k_{2}=k_{r} \sin \theta$ and $k_{r}^{2}+k_{3}^{2}=|k|^{2}$. From the relationship between the transform of the velocity and vorticity correlations $\Phi i j$ and $\Omega_{i j}$, we obtain

$$
\Omega_{i j}(\boldsymbol{k})=\Phi_{q q}(\boldsymbol{k})\left(|k|^{2} \delta_{i j}-k_{i} k_{j}\right)-|k|^{2} \Phi_{j i},
$$

which allows the two-dimensional velocity spectrum to be expressed in terms of the vorticity correlation transform

$$
E_{i j}^{2 D}\left(k_{r}\right)=\frac{k_{r}}{2} \int_{0}^{\infty} \int_{0}^{2 \pi} \frac{1}{|k|^{2}}\left(\Omega_{q q}(\boldsymbol{k})\left(\delta_{i j}-\frac{k_{i} k_{j}}{|k|^{2}}\right)-\Omega_{j i}(\boldsymbol{k})\right) \mathrm{d} \theta \mathrm{d} k_{3} .
$$

Using the inverse Fourier transform of (6.2) and the alternative expression for $W_{i j}$, (6.3), in (6.6), the two-dimensional energy tensor $E_{i j}^{2 D}$ leads, by integration of resulting delta functions, to 


$$
\begin{aligned}
& E_{i j}^{2 D}\left(k_{r}\right) \\
& =\frac{k_{r}}{\mathscr{V}} \sum_{m} l_{m} \int_{0}^{2 \pi} \int_{0}^{\pi} \int_{k_{r}}^{\left|k_{r} / \cos \alpha^{\prime}\right|} \int_{0}^{2 \pi} \frac{|\omega|^{2}}{\kappa} \frac{\left(\delta_{i j}-k_{i} k_{j} \kappa^{-2}-U_{3 i} U_{3 j}\right) \mathscr{P}\left(\alpha^{\prime}, \beta^{\prime}\right)}{\left(\kappa^{2}-k_{r}^{2}\right)^{1 / 2}\left(k_{r}^{2}-\kappa^{2} \cos ^{2} \alpha^{\prime}\right)^{1 / 2}} \mathrm{~d} \alpha^{\prime} \mathrm{d} \beta^{\prime} \mathrm{d} \kappa,
\end{aligned}
$$

where $\kappa^{2}$ is found to be equivalent to $k^{2}$, and $\mathscr{P}$ has been assumed to be independent of the spin angle $\gamma^{\prime}$. The two-dimensionally shell-summed energy spectrum $E_{i i}$ can now be related to the three-dimensionally shell-summed energy spectrum by using the sum identity $U_{3 i} U_{3 i}=1$ and the result (Lundgren 1982) that the shell-summed energy spectrum is related to the transform of the vorticity for an ensemble of vortices by

$$
E(\kappa)=\frac{2 \pi^{2}}{\mathscr{V}} \sum_{m} l_{m} \int_{0}^{2 \pi} \frac{1}{\kappa}\left|\hat{\omega}^{m}\left(\kappa_{1}, \kappa_{2}, t\right)\right|^{2} \mathrm{~d} \theta
$$

to arrive at

$$
E_{q q}^{2 D}\left(k_{r}\right)=\frac{k_{r}}{2 \pi^{2}} \int_{0}^{2 \pi} \int_{0}^{\pi} \int_{k_{r}}^{\left|k_{r} / \cos \alpha^{\prime}\right|} \frac{E(\kappa) \mathscr{P}\left(\alpha^{\prime}, \beta^{\prime}\right) \sin \alpha^{\prime}}{\left(\kappa^{2}-k_{r}^{2}\right)^{1 / 2}\left(k_{r}^{2}-\kappa^{2} \cos ^{2} \alpha^{\prime}\right)^{1 / 2}} \mathrm{~d} \alpha^{\prime} \mathrm{d} \beta^{\prime} \mathrm{d} \kappa .
$$

For the simple vortex alignment model used in the stretched vortex model, the p.d.f. has the form

which results in

$$
\mathscr{P}\left(\alpha^{\prime}, \beta^{\prime}\right)=\frac{4 \pi}{\sin \alpha_{o}} \delta\left(\alpha^{\prime}-\alpha_{o}\right) \delta\left(\beta^{\prime}-\beta_{o}\right)
$$

$$
E_{q q}^{2 D}\left(k_{r}\right)=\frac{2 k_{r}}{\pi} \int_{k_{r}}^{\left|k_{r} / \cos \alpha_{o}\right|} \frac{E(\kappa)}{\left(\kappa^{2}-k_{r}^{2}\right)^{1 / 2}\left(k_{r}^{2}-\kappa^{2} \cos ^{2} \alpha_{o}\right)^{1 / 2}} \mathrm{~d} \kappa .
$$

The component of the shell-summed energy spectra that results from the out-of-plane direction $E_{33}^{2 D}$ may be calculated directly by using the relation $k_{3}^{2}=k^{2}-k_{r}^{2}$ and the fact that $U_{33}=\cos \alpha^{\prime}$, giving

$$
E_{33}^{2 D}\left(k_{r}\right)=\frac{2 k_{r}}{\pi} \int_{k_{r}}^{\left|k_{r} / \cos \alpha_{o}\right|} \frac{\left(k_{r}^{2}-\kappa^{2} \cos ^{2} \alpha_{o}\right)^{1 / 2} E(\kappa)}{\kappa^{2}\left(\kappa^{2}-k_{r}^{2}\right)^{1 / 2}} \mathrm{~d} \kappa .
$$

At an instant in time, the velocity spectra for the resolved fields may be calculated as radial autocorrelations in a given plane of constant $x$, exploiting the periodic boundary conditions of the flow. The subgrid continuation of the velocity spectra is obtained by averaging the two-dimensional subgrid spectra over all the cells of the plane and the two-dimensional velocity spectra is in turn related to the known threedimensional spectra (3.12) for a given vortex by (6.11). The spectra of the velocity component normal to the plane (the $u$-velocity) is given by (6.12). In turn, this implies that the remaining two velocity components $v, w$, whose directions lie in the plane, contribute $E_{v}^{2 D}+E_{w}^{2 D}=E^{2 D}-E_{u}^{2 D}$. Such spectral continuations allow the presentation of anisotropic and isotropic portions of the total (resolved + subgrid) velocity spectra, as will be shown in $\S 7.1$.

\section{Subgrid continuation statistics}

We use the subgrid continuation ideas to develop consistent estimates of subgrid spectra for the velocity and scalar fields. In this context, the results show that the 

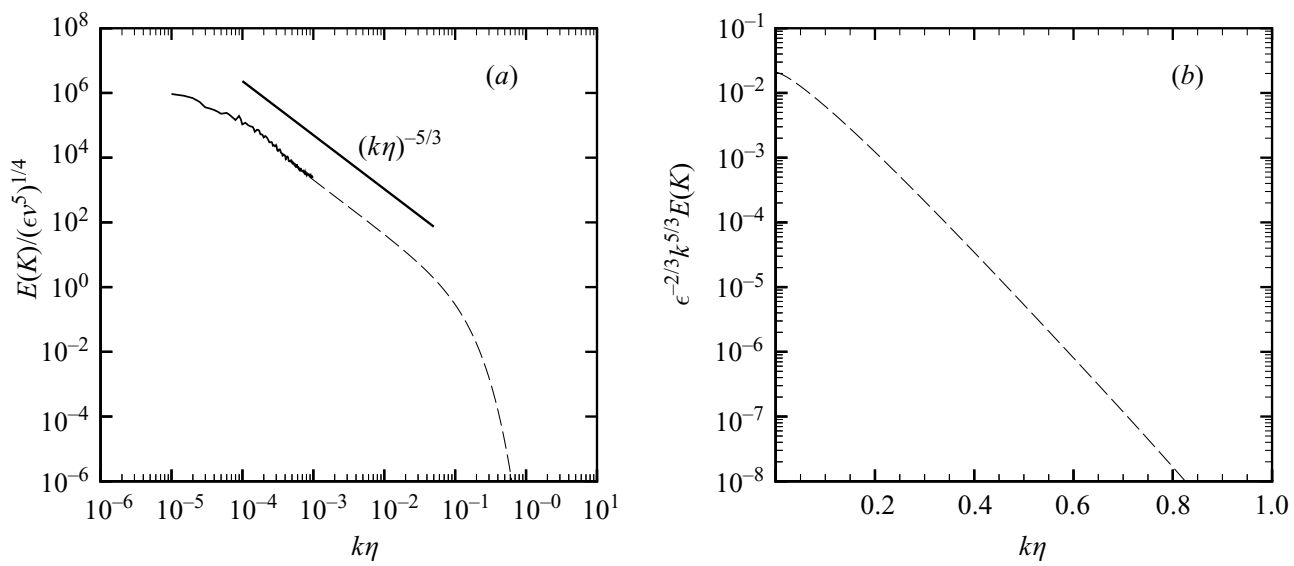

FIGURE 16. The radial spectra (solid line) with computed subgrid continuation (broken line) at time $t=10 \mathrm{~ms}$ computed in the centreplane of the TMZ. (a) Normalized spectra; (b) compensated spectra.

resolved spectra can be extended consistently into the subgrid. In the case of a mixing scalar, the continued spectra can incorporate any effects owing to non-unity Schmidt number, $S c=v / D$. Then, appropriate integration of the spectra generates estimates of subgrid scalar moments. In turn, these scalar moments can be used to construct assumed subgrid p.d.f.s of the mixing field of any reasonable complexity. For example, the resolved scalar field and second-order moment (variance) can be used to construct simple subgrid presumed p.d.f.s (Gaussian, beta, etc.). Presumed p.d.f.s with three or more moments can also be constructed with some additional modelling (Effelsberg \& Peters 1983; Mellado, Sarkar \& Pantano 2003).

\subsection{Turbulence spectra}

In the general practice of LES, it is not possible to obtain estimates of the full turbulence energy spectra, and consequently there is limited information to judge whether the resolved cutoff scale of the simulation reaches well within the inertial subrange (a theoretical premise of LES). Estimates of the complete turbulence spectra also enable more accurate calculation of the turbulent dissipation and hence, of the Kolmogorov length scale, $\eta=\left(v^{3} / \epsilon\right)^{1 / 4}$. With this in mind, our subgrid model and resolved geometry were chosen to allow such estimates to be made. Since the flow possesses two homogeneous directions, an averaged $\eta$ for each plane of constant $x$ can be obtained by using the plane-averaged values of $v$. Figure 16(a) shows the normalized and figure $16(b)$ the compensated resolved and continued turbulence spectra in the plane of constant $x$ at the middle of the mixing zone for $t=10 \mathrm{~ms}$. As expected, the roll-off for the spectra begins near $k \eta=0.1$, consistent with the majority of the dissipation occurring in the scales $k \eta>0.1$. Note that this estimate is not trivial. From (3.12), it is seen that the viscous decay of the spectrum of the stretched-spiral vortex is Gaussian, a natural consequence of the second-order viscous operator in the Navier-Stokes equations. In contrast, figure $16(b)$ indicates that the present estimate of the plane-averaged subgrid energy spectrum decays exponentially when $k \eta=O(1)$, in agreement with experimental evidence, Saddoughi \& Veeravalli (1994). The change from local Gaussian (in a cell) to plane-averaged exponential decay is explained by the influence of the statistics of fluctuating axial strain along the subgrid vortex axis (the quantity $|\tilde{a}|$ in (3.12)); for analysis, see Pullin \& Saffman (1993). 

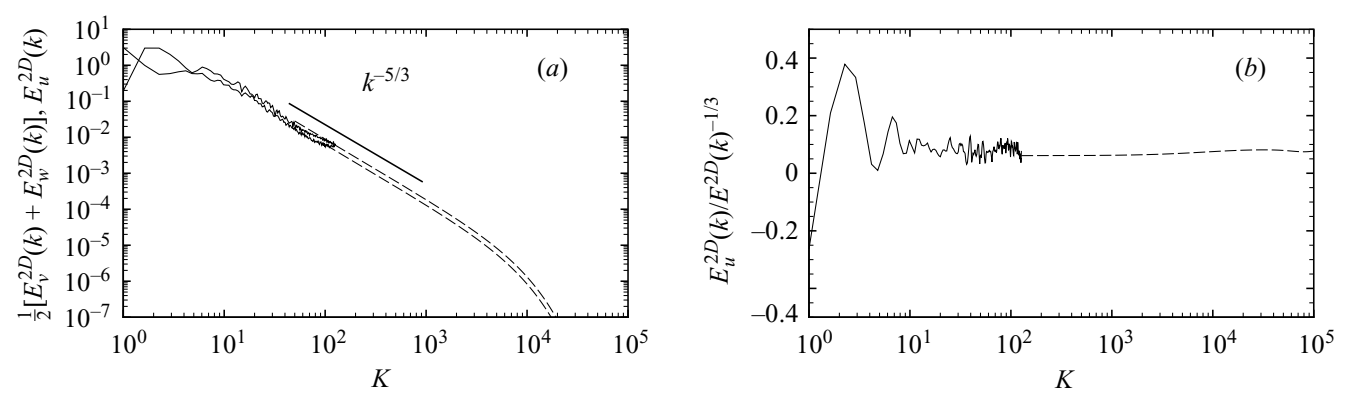

Figure 17. Components of the radial spectra at time $t=10 \mathrm{~ms}$ computed in the centreplane of the TMZ displaying resolved scale (solid line) and continuation (broken line). In $(a)$ the spectra is divided into the anisotropic $E_{u}^{2 D}(k)$ (upper curve) and isotropic $(1 / 2)\left[E_{v}^{2 D}(k)+E_{w}^{2 D}(k)\right]$ (lower curve) directions. In $(b)$ a measure of the anisotropy is displayed as $E_{u}^{2 D}(k) / E^{2 D}(k)-1 / 3$.

As discussed in the previous section, it is now possible to use the stretched-vortex model to derive estimates of the isotropic and anisotropic parts of the spectra with the present multiscale approach. Figure 17( $a$ ) shows the anisotropic, $u$ component of velocity $\mathrm{E}_{u}^{2 D}$, and the isotropic radial spectra of velocity components, $\mathrm{E}_{v}^{2 D}+\mathrm{E}_{w}^{2 D}$. In this figure, we show the spectra obtained from the resolved velocity fields (continuous line) as well as the subgrid continuation part (broken lines) at the same location used in figure 16. The continued spectra was obtained by computing the two-dimensional radial spectral, (6.11)-(6.12), for each cell in a plane and then averaging the result for the entire plane. For comparison, we also show the $-5 / 3$ constant slope line in the same figure. It can be noted that this LES is conducted at a resolution well within the inertial subrange, since we recover more than a decade of wavenumbers with a Kolmogorov-like spectra in the resolved field. Figure $17(b)$ gives a scaledependent measure of the anisotropy that the model continues into the subgrid. This indicates that $E_{u}^{2 D}$ contains, at almost every scale, more than a third of the total energy. The spectra detailed from the simulation is reported unprocessed, that is, all wavenumber contributions are shown and no filtering is performed at the highest wavenumbers. It is our experience that the use of skew-symmetric-type discretization together with bandwidth-optimized centred stencils, like the present TCD, leads to very good spectral results with minimal accumulation of energy owing to aliasing errors at the high-wavenumber end of the spectrum.

\subsection{Effect of Schmidt number on continued scalar spectra}

We remark from the outset that we will allow the ratio of molecular diffusivity to scalar diffusivity, known as the Schmidt number, to take a series of values that are different from the actual ratio corresponding to our gas mixture. This is done in the spirit of exemplifying the methodology for other gases with different diffusivities and is carried out in both this subsection and the following one.

Pullin \& Lundgren (2001) presented an approximate solution to the equations describing the mixing of a passive scalar inside a stretched-spiral vortex. They obtained the scalar spectrum (their equations (99)-(106)), which, for the scalar $\psi$, we express in the form

$$
E_{\psi}(k)=K_{\psi}\left(k^{-5 / 3} \exp \left(-\frac{(4 v+2 D) k^{2}}{3 \tilde{a}}\right)+\frac{8}{5 \pi}\left(\frac{2 \Gamma}{\tilde{a}}\right)^{1 / 3} k^{-1} \exp \left(-\frac{2 D k^{2}}{3 \tilde{a}}\right)\right),
$$




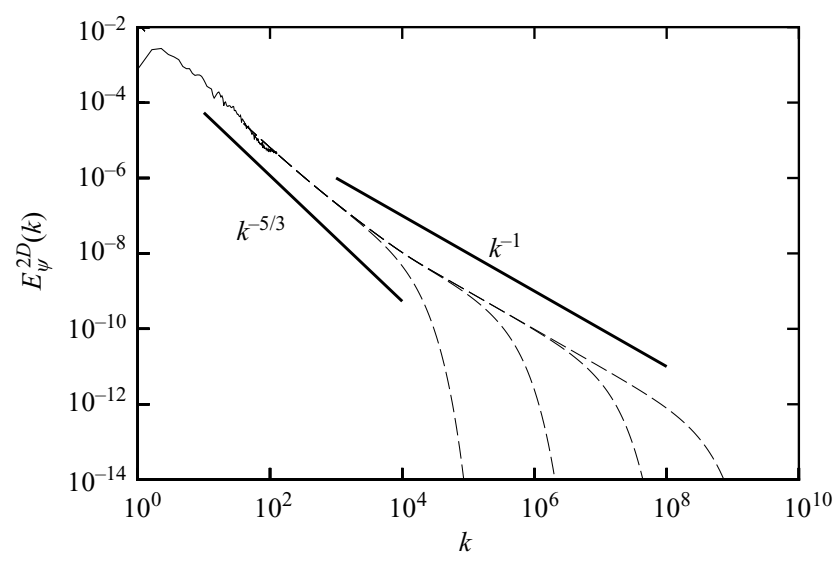

FIGURE 18. The time $t=10 \mathrm{~ms}$ radial spectra of the scalar computed in the centre plane of the TMZ with subgrid continuation (broken lines) assuming, from left to right, $S c=1,10^{3}$, $10^{6}$, and $10^{9}$.

where $\tilde{a}$ is the axial resolved-scale strain rate along the subgrid vortex axis, $\Gamma$ is the subgrid vortex circulation and $K_{\psi}$ is a group prefactor whose numerical value depends on physical quantities that describe the internal structure of the vortex and the initial scalar field. Equation (7.1) is consistent with phenomenological models for scalar mixing in turbulence (see Tennekes \& Lumley 1974). When the scalar diffusivity $D \ll v$, there is a rapid decay like $k^{-5 / 3}$ through the inertial convective range, with slower decay as $k^{-1}$ before reaching diffusive cutoff at the Batchelor scale, while for diffusivities $D=O(v)$ the cutoff occurs before the $k^{-1}$ range begins. The derivation of this form is consistent with the subgrid scalar flux $(3.3 d)$; the mixture fraction within a computational cell is imagined to be wound by an elemental Lundgren subgrid vortex. The group prefactor $K_{\psi}$ is computed here for each cell by dynamic structure function matching, similar to that outlined in $\S 3.2$ for the subgrid velocity spectrum. This spherically averaged structure function formed by $\psi$ evaluated from the resolved scale at the length scale $\Delta=\Delta x, \tilde{\mathscr{F}}_{2}^{\psi}(\Delta)$, is equated with the subgrid expression of the same quantity

$$
\tilde{\mathscr{F}}_{2} \psi(\Delta)=4 K_{\psi} \Delta^{2 / 3} \int_{0}^{\pi}\left(s^{-5 / 3}+\frac{8}{5 \pi}\left(\frac{2 \Gamma}{\tilde{a} \Delta^{2}}\right)^{1 / 3} s^{-1}\right)\left(1-\frac{\sin s}{s}\right) \mathrm{d} s .
$$

For our model, the remaining parameter is the subgrid circulation $\Gamma / v$, which, from (7.1), largely determines the relative weighting between the $k^{-5 / 3}$ and the $k^{-1}$ spectral components. Pullin \& Lundgren (2001) found that $\Gamma / v=1000$ gave fair agreement with experimental data at $S c>1$, and we adopt this value here. This is the only additional assumption required for the subgrid continuation of the scalar spectrum. It may seem paradoxical that $S c$ dependence at small scales can be inferred from LES in which $S c$ does not appear explicitly. This is because we implicitly assume, in the LES, that the resolved scale cutoff $\Delta_{c}$ lies somewhere in the inertial range, and that, consequently, the flux of scalar variance off the resolved-scale grid is independent of $S c$ for $S c \geqslant 1$. This is consistent with the hypothesis that mixing is an essentially small-scale process that is subgrid in the present LES.

Figure 18 shows continuation of the resolved scale composition spectra into the subgrid for different Schmidt numbers. As in $§ 7.1$, this was done by computing 
the two-dimensional radial spectral continuation for each cell in a plane and then averaging the result for the entire plane. As can be seen, the large $S c$ spectra display both the $k^{-5 / 3}$ and $k^{-1}$ range. While the tails of the spectra can be quite long for this higher Schmidt number, its contribution to the subgrid scalar variance is typically not large, unless the Schmidt number is huge.

The subgrid variance of the composition scalar $\sigma_{\psi}^{2}$ within a given cell may be computed from the model

$$
\sigma_{\psi}^{2}=2 \int_{k_{c}}^{\infty} E_{\psi}(k) \mathrm{d} k,
$$

where $k_{c}$ is the cutoff wavelength associated with the grid spacing and the results from the structure function matching, which implicitly assumes $2 \int_{0}^{\infty} E_{\psi} \mathrm{d} k=\mathscr{V}^{-1} \int|\psi|^{2} \mathrm{~d} \boldsymbol{x}$. By examining the relevant portion of (7.1), it is easy to see that there is only logarithmic dependence on Schmidt number for the subgrid contribution to the total variance, that is

$$
\begin{aligned}
\int_{k_{c}}^{\infty} s^{-1} \exp \left(-\frac{2 D s^{2}}{3 \tilde{a}}\right) \mathrm{d} s & =\frac{1}{2} \int_{D k_{c}^{2} 2 /(3 \tilde{a})}^{\infty} s^{-1} \exp (-s) \mathrm{d} s, \\
& =-\gamma-\log (\xi)-\sum_{n=1}^{\infty}(-1)^{n} \frac{\xi^{n}}{n(n !)},
\end{aligned}
$$

where $\xi=D k_{c}^{2} 2 /(3 \tilde{a})$ and $\gamma=\lim _{\xi \rightarrow 0^{+}}\left(\ln \xi^{-1}-\int_{\xi}^{\infty} s^{-1} \exp (-s) \mathrm{d} s\right) \sim 0.5772$ is Euler's constant. For fixed $v$ and large $S c$ then, it can be seen that the leading behaviour is $\log (S c)-\log \left(2 v k_{c}^{2} /(3 \tilde{a})\right)-\gamma$. This result is also consistent with the arguments presented by Dimotakis (1989) that account for the Schmidt-number dependence of the total scalar variance.

\subsection{Effect of Schmidt number on continued p.d.f.}

Actual p.d.f.s (regular or subgrid) are not generally known in LES. They contain the complete single-point statistics of the state of the mixture. Two approaches are commonly used in LES to estimate or model the subgrid p.d.f.: solve a subgrid transport p.d.f. equation (Colucci et al. 1998) or directly presume a functional form with specified moments (Cook \& Riley 1994). Here, we take this latter approach for simplicity, but the alternative of solving a transported subgrid p.d.f. equation is also feasible. Using results from previous sections, it is now possible to construct a presumed subgrid p.d.f. that can take into account possible non-unity Schmidtnumber effects. We consider the subgrid Favre p.d.f. of mixture fraction, $\tilde{P}_{s g s}(\psi)$, to clarify ideas.

There are typically two uses of $\tilde{\mathscr{P}}_{\text {sgs }}$. In the first case, the physics of the problem are such that there is two-way coupling between the scalar and the flow and we must obtain subgrid expectations of some nonlinear function of $\psi$ for LES closure; let us call this function $f(\psi)$. Then, knowledge of $\tilde{\mathscr{P}}_{\text {sgs }}$ gives, by direct integration,

$$
f(\widetilde{\psi ; \boldsymbol{x}}, t)=\int f(\psi) \tilde{\mathscr{P}}_{s g s}(\psi ; \boldsymbol{x}, t) \mathrm{d} \psi .
$$

This is typically the closure of LES for combustion. In the second case, we may be interested in mixing per se. The question of interest now is whether we can estimate the total scalar p.d.f., $\widetilde{\mathrm{P}}(\psi)$, (resolved and subgrid) from $\tilde{\mathscr{P}}_{\text {sgs }}$. To expose the relationship between these two p.d.f.s, we must recall Gao \& O'Brien (1993) and introduce the fine-grained p.d.f., $\delta\left(\psi-\psi^{*}(\boldsymbol{x}, t)\right)$, where $\delta(x)$ is Dirac's delta function, $\psi$ is the sample 
space variable and $\psi^{*}(\boldsymbol{x}, t)$ is a realization of the random space-time varying field. We show the derivation of this relationship for the Reynolds and Favre p.d.f.s. The regular and subgrid p.d.f.s are obtained from the fine-grained p.d.f. through

$$
\begin{aligned}
\mathrm{P}(\psi ; \boldsymbol{x}, t) & =\left\langle\left\langle\delta\left(\psi-\psi^{*}(\boldsymbol{x}, t)\right)\right\rangle\right\rangle, \\
\mathscr{P}_{s g s}(\psi ; \boldsymbol{x}, t) & =\int G\left(\boldsymbol{x}-\boldsymbol{x}^{\prime}\right) \delta\left(\psi-\psi^{*}\left(\boldsymbol{x}^{\prime}, t\right)\right) \mathrm{d} x^{\prime},
\end{aligned}
$$

where $\langle\langle\cdot\rangle\rangle$ denotes ensemble averaging and $G(\boldsymbol{x})$ is the convolution kernel as in (3.2). Now, ensemble averaging (7.7), commuting the spatial and ensemble-averaging operators (both are linear operators), leads to

$$
\left\langle\left\langle\mathscr{P}_{s g s}\right\rangle\right\rangle=\left\langle\left\langle\int G\left(\boldsymbol{x}-\boldsymbol{x}^{\prime}\right) \delta\left(\psi-\psi^{*}\left(\boldsymbol{x}^{\prime}, t\right)\right) \mathrm{d} x^{\prime}\right\rangle\right\rangle=\int G\left(\boldsymbol{x}-\boldsymbol{x}^{\prime}\right) \mathrm{P}\left(\psi ; \boldsymbol{x}^{\prime}, t\right) \mathrm{d} x^{\prime}
$$

We further observe that: by construction, $G$ only affects the scales in the neighbourhood and below the subgrid cutoff scale $\Delta_{c}$ and that the spatial variations of $\mathrm{P}$ happen, in general, on scales of the order of the integral scale $\ell$. Then, we can take the p.d.f. outside the integral (using the normalization property of the filter kernel) giving

$$
\int G\left(\boldsymbol{x}-\boldsymbol{x}^{\prime}\right) \mathrm{P}\left(\psi ; \boldsymbol{x}^{\prime}, t\right) \mathrm{d} x^{\prime} \approx \mathrm{P}(\psi ; \boldsymbol{x}, t)
$$

The approximation in (7.9) is quite accurate because the error, that is at most of order $\Delta_{c} / \ell$, is small in LES.

The equivalent of (7.8) for the Favre version of the p.d.f. is

$$
\begin{aligned}
\left\langle\left\langle\bar{\rho} \tilde{\mathscr{P}}_{s g s}\right\rangle\right\rangle & =\int G\left(\boldsymbol{x}-\boldsymbol{x}^{\prime}\right)\left\langle\left\langle\rho^{*}\left(\boldsymbol{x}^{\prime}, t\right) \delta\left(\psi-\psi^{*}\left(\boldsymbol{x}^{\prime}, t\right)\right)\right\rangle\right\rangle \mathrm{d} x^{\prime} \\
& =\int G\left(\boldsymbol{x}-\boldsymbol{x}^{\prime}\right)\left\{\int \rho\left\langle\left\langle\delta\left(\rho-\rho^{*}\left(\boldsymbol{x}^{\prime}, t\right)\right) \delta\left(\psi-\psi^{*}\left(\boldsymbol{x}^{\prime}, t\right)\right)\right\rangle\right\rangle \mathrm{d} \rho\right\} \mathrm{d} x^{\prime} \\
& =\int G\left(\boldsymbol{x}-\boldsymbol{x}^{\prime}\right)\left\{\int \rho P\left(\rho, \psi ; \boldsymbol{x}^{\prime}, t\right) \mathrm{d} \rho\right\} \mathrm{d} x^{\prime} \\
& =\int G\left(\boldsymbol{x}-\boldsymbol{x}^{\prime}\right)\left\langle\left\langle\rho\left(\boldsymbol{x}^{\prime}, t\right)\right\rangle\right\rangle \tilde{\mathrm{P}}\left(\psi ; \boldsymbol{x}^{\prime}, t\right) \mathrm{d} x^{\prime} .
\end{aligned}
$$

In the last term, we have removed the superscript from the ensemble average of $\rho$, since there is no longer any ambiguity with the sample variable. Using the approximation introduced in (7.9) and neglecting the additional variable density contributions resulting from filtering the product of the ensemble-averaged density with the Favre p.d.f., we arrive at

$$
\int G\left(\boldsymbol{x}-\boldsymbol{x}^{\prime}\right)\left\langle\left\langle\rho\left(\boldsymbol{x}^{\prime}, t\right)\right\rangle\right\rangle \tilde{\mathrm{P}}\left(\psi ; \boldsymbol{x}^{\prime}, t\right) \mathrm{d} x^{\prime} \approx\langle\langle\rho(\boldsymbol{x}, t)\rangle\rangle \tilde{\mathrm{P}}(\psi ; \boldsymbol{x}, t) .
$$

To clarify ideas, we now introduce a concrete function $\tilde{\mathscr{P}}_{\text {sgs }}$. Among the possible choices of presumed p.d.f.s, the beta distribution is very popular (Cook \& Riley 1994). For this model, $\tilde{\mathscr{P}}_{\text {sgs }}(\psi)$ within a given cell is assumed to take the form

$$
\tilde{\mathscr{P}}_{\text {sgs }}(\psi ; \boldsymbol{x}, t)=\frac{\Gamma(a+b)}{\Gamma(a) \Gamma(b)} \psi^{a-1}(1-\psi)^{b-1},
$$




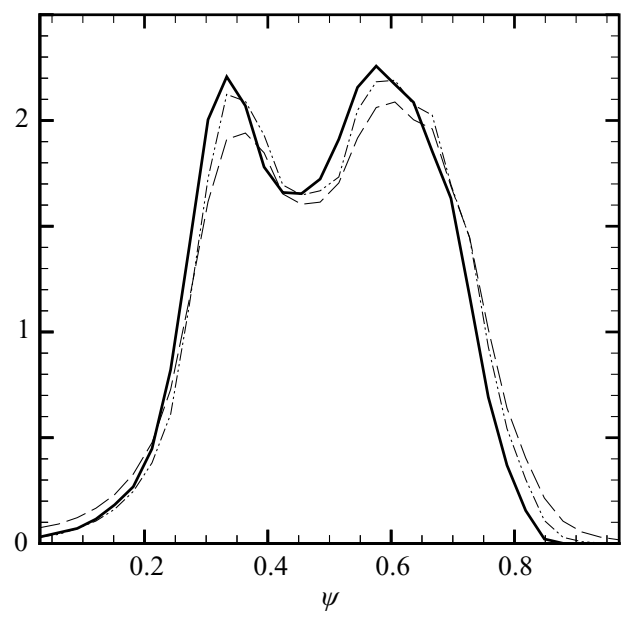

FIGURE 19. Effect of Schmidt number on continued p.d.f. of mixture fraction calculated in the centre of the mixing zone at $t=10 \mathrm{~ms}$ with resolved scale p.d.f (solid line) and values $S c=1$, $10^{7}$ (dash-dot and broken line, respectively).

where $\Gamma(x)$ is the gamma function. The parameters $a$ and $b$ are determined by evaluating the first- and second-order moments of $\tilde{\mathscr{P}}_{s g s}$, leading to the following relationships with the resolved scales: $a=\tilde{\psi}\left[\tilde{\psi}(1-\tilde{\psi}) / \sigma_{\psi}^{2}-1\right]$ and $b=a(1 / \tilde{\psi}-1)$. Recall, the LES gives $\tilde{\psi}$ directly, and the scalar variance can be estimated from (7.3). For clarity, we avoided denoting the dependence of $a$ and $b$ on space and time explicitly, but note that $\tilde{\mathscr{P}}_{s g s}$ varies with position and time parametrically through the dependence of $a$ and $b$ on the resolved scalar and scalar variance fields.

The formal method for computing the estimate for $\tilde{\mathrm{P}}$ in the mixing zone consist in noticing that (7.12) is in reality the subgrid conditional-p.d.f. $\tilde{P}_{s g s}\left(\psi \mid \tilde{\psi}, \sigma_{\psi}^{2}\right)$. Using Bayes theorem, the subgrid joint-p.d.f. is now given by

$$
\tilde{\mathscr{P}}_{s g s}\left(\psi, \tilde{\psi}, \sigma_{\psi}^{2} ; \boldsymbol{x}, t\right)=\tilde{\mathscr{P}}_{s g s}\left(\psi \mid \tilde{\psi}, \sigma_{\psi}^{2}\right) \tilde{\mathscr{P}}_{s g s}\left(\tilde{\psi}, \sigma_{\psi}^{2} ; \boldsymbol{x}, t\right),
$$

and the subgrid p.d.f. of $\psi$ is given by

$$
\tilde{\mathscr{P}}_{s g s}(\psi ; \boldsymbol{x}, t)=\iint \tilde{\mathscr{P}}_{s g s}\left(\psi \mid \tilde{\psi}, \sigma_{\psi}^{2}\right) \tilde{\mathscr{P}}_{s g s}\left(\tilde{\psi}, \sigma_{\psi}^{2} ; \boldsymbol{x}, t\right) \mathrm{d} \tilde{\psi} \mathrm{d} \sigma_{\psi}^{2}
$$

Then, $\tilde{\mathrm{P}}$ results from the integration and ensemble averaging given by (7.11), that for our flow is expressed as the planar-averaged expression

$$
\tilde{\mathrm{P}}(\psi ; \boldsymbol{x}, t) \simeq \frac{\left\langle\bar{\rho} \tilde{\mathscr{P}}_{s g s}(\psi ; \boldsymbol{x}, t)\right\rangle}{\langle\bar{\rho}(\boldsymbol{x}, t)\rangle},
$$

where $\langle\langle\rho(\boldsymbol{x}, t)\rangle\rangle$ has been approximated by $\langle\bar{\rho}(\boldsymbol{x}, t)\rangle$. In practice, these steps are performed simultaneously by computing the average of the conditional-p.d.f., $\mathscr{P}_{s g s}\left(\psi \mid \tilde{\psi}, \sigma_{\psi}^{2}\right)$, across the cells of the plane in question, as shown by Jiménez et al. (1997) for the constant density case.

Figure 19 shows the effect of a range of Schmidt numbers on the estimate of the total p.d.f. taken in the centre of the mixing zone at one time. It can be seen that as the Schmidt number increases, the logarithmic dependence of the variance on the Schmidt number results in relatively small, but still visible, changes in the overall 
p.d.f. This is because the subgrid cutoff scale is well within the inertial subrange in our simulation so that the contribution of the subgrid scalar variance is small with respect to the resolved counterpart. Note also that the effect of Schmidt number is not monotone. Some regions of the p.d.f. are lifted with increasing Schmidt number while others sink; but, in general, the qualitative shape of the p.d.f. is not altered by the inclusion of subgrid effects.

In $\S 5.4$, we postponed the discussion of the effect of the subgrid scales on the p.d.f. of mixture fraction and contented ourselves with the statistics obtained from the resolved scales. At this point, we can now consider the consequences of neglecting the effect of the unresolved scales within the context of the results of our modelling. Evidently, most of the scalar variance is already represented by the resolved field and only a small amount, that could become non-negligible if the Schmidt number is huge, is present in the subgrid. We can now go back to figure 15 and claim with a degree of certainty that those p.d.f.s are representative of the state of the mixture. Nevertheless, we would like to remark that this statement is based on an estimate of the effect of the subgrid scales on the p.d.f. using additional modelling, the actual p.d.f. is lost when LES modelling is introduced.

\section{Conclusions}

The Richtmyer-Meshkov instability with reshock was studied in the canonical geometry of a rectangular shock-tube with a square cross-section, the gases in the simulation and the geometry of the domain, and the strength of the incident shock were chosen to match those of the experiments of Vetter \& Sturtevant (1995). The high Reynolds numbers produced within the mixing zone between gases required the use of the techniques of large-eddy simulation (LES), and the stretched-vortex subgrid model was employed. A specially constructed hybrid numerical method was used, one that is conservative and shock capturing, but reverts to a numerically dissipationless form away from shocks. Of the three different experiments simulated, all had good agreement with the observed growth rates of the mixing zone after the initial shock and after reshock. The actual width of the mixing zone was not expected to compare well with the observed values owing to the large degree in uncertainty in the initial conditions, but it was found that the width at the end of the simulation times were between $4 \%$ and $10 \%$ of the observed values.

Examination of the turbulent kinetic energy indicated that an expansion wave which follows reshock plays a major role, comparable to that of the reshock, in driving the growth of the mixing layer. In general, the flow in the mixing zone is only weakly compressible, but the energy spectrum indicates that an inertial subrange does not fully develop until after the passage of this expansion wave. During this turbulent decay period, the subgrid dissipation resulting from the stretched vortex model allowed for the calculation of a Reynold number and a related outer length scale. Furthermore, the Kolmogorov scale and the subgrid continued spectra were calculated for this flow. Additional modelling gave insight into the dependence of the mixing of the constituent gases on the Schmidt number. This dependence was shown to be only logarithmic, but still had a measurable effect.

This work was supported by the Advanced Simulation and Computing (ASC) Program under subcontract no. B341492 of DOE contract W-7405-ENG-48. The authors would like to acknowledge the many helpful conversations with P.E. Dimotakis and D. I. Meiron. 


\section{REFERENCES}

BILGER, R. W. 1977 Comment on 'Structure of turbulent shear flows: A new look'. AIAA J. 15, 1056.

BLAisDell, G. A. 1991 Numerical simulation of compressible homogeneous turbulence. PhD thesis, Stanford University.

Brouillette, M. 2002 The Richtmyer-Meshkov instability. Annu. Rev. Fluid Mech. 34, 445-468.

Cohen, R. H., Dannevik, W. P., Dimits, A. M., Eliason, D. E., Mirin, A. A., Zhou, Y., Porter, D. H. \& Woodward, P. R. 2002 Three-dimensional simulation of a Richtmyer-Meshkov instability with a two-scale initial perturbation. Phys. Fluids 14, 3692-3709.

Colucci, P. J., Jaberi, F. A., Givi, P. \& Pope, S. B. 1998 Filtered density function for large eddy simulation of turbulent reacting flows. Phys. Fluids 10, 499.

Cook, A. W. \& Riley, J. J. 1994 A subgrid model for equilibrium chemistry in turbulent flows. Phys. Fluids 6, 2868.

Dimotakis, P. E. 1989 Turbulent shear layer mixing with fast chemical reactions. In Turbulent Reactive Flows. Lecture Notes in Engineering, vol. 40, pp. 417-485. Springer.

Ducros, F., Laporte, F., Souleres, T., Guinot, V., Moinat, P. \& Caruelle, B. 2000 High-order fluxes for conservative skew-symmetric-like schemes in structured meshes: Application to compressible flows. J. Comput. Phys. 161, 114-139.

Effelsberg, E. \& Peters, N. 1983 A composite model for the conserved scalar p.d.f. Combust. Flame 50, 351.

Gao, F. \& O’Brien, E. E. 1993 A large-eddy simulation scheme for turbulent reacting flows. Phys. Fluids A 5, 1282.

GHosal, S. 1996 An analysis of numerical errors in large-eddy simulations of turbulence. J. Comput. Phys. 125, 187-206.

Ghosal, S. 1999 Mathematical and physical constraints on large-eddy simulation of turbulence. AIAA J. 37, 425-433.

Gottlieb, S., Shu, C. W. \& Tadmor, E. 2001 Strong stability-preserving high-order time discretization methods. SIAM Rev. 43, 89-112.

Greenough, J. A. \& Burke, E. 2004 The effect of initial conditions on late time asymptotics and mixing for multimode Richtmyer-Meshov instability. In 9th Intl Workshop on the Physics of Compressible Turbulent Mixing, 19-23 July, Cambridge, UK.

Hill, D. J. \& Pullin, D. I. 2004 Hybrid tuned center-difference-WENO method for large eddy simulations in the presence of strong shocks. J. Comput. Phys. 194, 435-450.

Honein, A. E. \& Moin, P. 2004 Higher entropy conservation and numerical stability of compressible turbulence simulations. J. Comput. Phys. 201, 531-545.

Jiang, G. S. \& ShU, C. W. 1996 Efficient implementation of weighted ENO schemes. J. Comput. Phys. 126, 202-228.

Jiménez, J., Liñán, A. L., Rogers, M. M. \& Higuera, F. J. 1997 A priori testing of subgrid models for chemically reacting non-premixed turbulent shear flows. J. Fluid Mech. 349, $149-171$.

Kosovic, B., Pullin, D. I. \& Samtaney, R. 2002 Subgrid-scale modeling for large-eddy simulations of compressible turbulence. Phys. Fluids 14, 1511-1522.

Lesieur, M. \& Metais, O. 1996 New trends in large-eddy simulations of turbulence. Annu. Rev. Fluid Mech. 28, 45-82.

Linde, J. D., McCrory, R. L. \& CAmpbell, E. M. 1992 Progress toward ignition and burn propagation in inertial confinement fusion. Phys. Today 45, 32-40.

Lundgren, T. S. 1982 Strained spiral vortex model for turbulent fine-structure. Phys. Fluids 25, 2193-2203.

Maran, S. P., Sonneborn, G., Pun, C. S. J., Lundqvist, P., Iping, R. C. \& Gull, T. R. 2000 Physical conditions in circumstellar gas surrounding SN 1987A 12 years after outburst. Astrophys. $J$. 545, 390-398.

Mattner, T. W., Pullin, D. I. \& Dimotakis, P. E. 2004 Large eddy simulations of miscible Rayleigh-Taylor instability. In 9th Intl Workshop on the Physics of Compressible Turbulent Mixing, 19-23 July, Cambridge, UK.

Mellado, J. P., Sarkar, S. \& Pantano, C. 2003 Reconstruction subgrid models for nonpremixed combustion. Phys. Fluids 15, 3280-3307. 
Meshkov, E. E. 1969 Instability of the interface of two gases accelerated by a shock wave. Sov. Fluid Dyn. 4, 101-108.

Mikaelian, K. O. 1989 Turbulent mixing generated by Rayleigh-Taylor and Richtmyer-Meshkov instabilities. Physica D 36, 343-357.

MisRa, A. \& Pullin, D. I. 1997 A vortex-based subgrid stress model for large-eddy simulation. Phys. Fluids 9, 2443-2454.

Pantano, C., Deiterding, R., Hill, D. J. \& Pullin, D. I. 2005 A low-numerical dissipation patchbased adaptive mesh refinement method for large-eddy simulation of compressible flows. J. Comput. Phys. (under review).

Pope, S. B. 2004 Ten questions concerning the large-eddy simulation of turbulent flows. N. J. Phys. 6, art. 35 .

Prasad, J. K., Rasheed, A., Kumar, S. \& Sturtevant, B. 2000 The late-time development of the Richtmyer-Meshkov instability. Phys. Fluids 12, 2108-2115.

Pullin, D. I. 2000 A vortex-based model for the subgrid flux of a passive scalar. Phys. Fluids 12, 2311-2319.

Pullin, D. I. \& Lundgren, T. S. 2001 Axial motion and scalar transport in stretched spiral vortices. Phys. Fluids 13, 2553-2563.

Pullin, D. I. \& Saffman, P. G. 1993 On the Lundgren-Townsend model of turbulent fine scales. Phys. Fluids A 5, 126-145.

Pullin, D. I. \& Saffman, P. G. 1994 Reynolds stresses and one-dimensional spectra for a vortex model of homogeneous anisotropic turbulence. Phys. Fluids 6, 1787-1796.

Reid, R. C., Pransuitz, J. M. \& Poling, B. E. 1987 The Properties of Gases and Liquids. McGrawHill.

Richtmyer, R. D. 1960 Taylor instability in shock acceleration of compressible fluids. Commun. Pure Appl. Maths 13, 297-319.

Saddoughi, S. G. \& Veeravalli, S. V. 1994 Local isotropy in turbulent boundary-layers at high Reynolds numbers. J. Fluid Mech. 268, 333-372.

Smarr, L., Wilson, J. R., Barton, R. T. \& Bowers, R. L. 1981 Rayleigh-Taylor overturn in super-nova core collapse. Astrophys. J. 246, 515-525.

Sonneborn, G., Pun, C. S. J., Garnavich, P. \& Kirshner, R. 1999 Birth of supernova remnant 1987a. IAU Symp. pp. 136-138.

Tennekes, H. \& Lumley, J. L. 1974 A First Course in Turbulence. MIT Press, Cambridge, MA.

Thompson, K. W. 1987 Time dependent boundary conditions for hyperbolic systems. J. Comput. Phys. 68, 1-24.

Vetter, M. \& Sturtevant, B. 1995 Experiments on the Richtmyer-Meshkov instability of an air/SF 6 interface. Shock Waves 4, 247-252.

Voelkl, T., Pullin, D. I. \& Chan, D. C. 2000 A physical-space version of the stretched-vortex subgrid-stress model for large-eddy simulation. Phys. Fluids 12, 1810-1825.

YAnG, J., Kubota, T. \& Zukoski, E. E. 1993 Applications of shock-induced mixing to supersonic combustion. AIAA J. 31, 854-862.

ZABUSKY, N. J. 1999 Vortex paradigm for accelerated inhomogeneous flows: Visiometrics for the Rayleigh-Taylor and Richtmyer-Meshkov environments. Annu. Rev. Fluid Mech. 31, 495-536.

Zang, T. A., Dahlburg, R. B. \& Dahlburg, J. P. 1992 Direct and large-eddy simulations of 3-dimensional compressible Navier-Stokes turbulence. Phys. Fluids A-Fluid Dyn. 4, 127-140. 